\title{
Synthesis of oligosaccharide derivatives related to those from sanqi, a Chinese herbal medicine from Panax notoginseng
}

\author{
Feng Yang, Yuguo Du* \\ Research Center for Eco-Environmental Sciences, Academia Sinica, PO Box 2871, Beijing 100085, PR China
}

Received 16 November 2001; accepted 4 January 2002

\begin{abstract}
Oligosaccharide derivatives from sanqi, a Chinese herbal medicine derived from Panax notoginseng, methyl $\beta$-D-galactopyran-

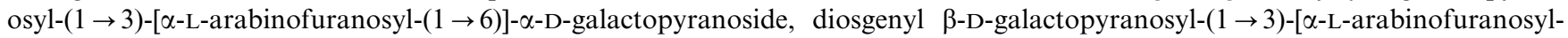
$(1 \rightarrow 6)]-\alpha$-D-galactopyranoside, and methyl $\beta$-D-galactopyranosyl-( $1 \rightarrow 3)-[\alpha$-L-arabinofuranosyl- $(1 \rightarrow 6)]-\alpha$-D-galactopyranosyl$(1 \rightarrow 4)$ - $\beta$-D-galactopyranosyl-( $(1 \rightarrow 3)$-[ $\alpha$-L-arabinofuranosyl- $(1 \rightarrow 6)]-\alpha$-D-galactopyranoside, were synthesized under standard glycosylation conditions. An unexpected $\alpha-(1 \rightarrow 4)$ linkage was formed predominantly in the presence of neighboring participation group during regioselective synthesis of hexasaccharide via $3+3$ strategy. (c) 2002 Elsevier Science Ltd. All rights reserved.
\end{abstract}

Keywords: Natural products; Neighboring-group effects; Stereoselectivity; Glycosylations; Oligosaccharide

\section{Introduction}

Panax notoginseng (Burk.) F.H. Chen. (sanqi) is widely used in China as a medicine to promote the circulation of blood. ${ }^{1}$ It is very effective in treating coronary heart diseases and angina pectoris. The active components in sanqi are believed to be carbohydrates and their saponins. ${ }^{2}$ Structural analysis shows that $\beta$-Dgalactopyranosyl- $(1 \rightarrow 3)$-[ $\alpha$-L-arabinofuranosyl- $(1 \rightarrow 6)$ ]$\mathrm{D}$-galactopyranose is the major fraction in active sanqi oligosaccharides. ${ }^{3}$ In stereocontrolled glycosylation, the best-established method is based on the participation of a group at C-2 of the glycosyl donor to direct 1,2-trans glycosidic bond formation. ${ }^{4}$ Although the experimental conditions for this procedure have been modified case by case, the basic principle of neighboring-group participation has not been obliterated. ${ }^{5}$ We herein present the synthesis of sanqi oligosacchride derivatives and an unexpected result in regio- and stereoselective synthesis of a hexasaccharide in the presence of neighboringgroup participation during a $3+3$ glycosylation.

\footnotetext{
* Corresponding author. Tel.: + 86-10-62914475; fax: + 86-10-62923563.

E-mail address: ygdu@mail.rcees.ac.cn (Y. Du).
}

\section{Results and discussion}

Regioselective glycosylation of 2,3,5-tri- $O$-benzoyl- $\alpha$ L-arabinofuranosyl trichloroacetimidate ${ }^{6} \quad$ (1) and methyl 3,4-O-isopropylidene- $\alpha$-D-galactopyranoside ${ }^{7}$ (2) in dry $\mathrm{CH}_{2} \mathrm{Cl}_{2}$ with TMSOTf as a promoter afforded methyl 2,3,5-tri- $O$-benzoyl- $\alpha$-L-arabinofuranosyl-( $1 \rightarrow 6)$-3,4- $O$-isopropylidene- $\alpha$-D-galactopyranoside $(3,76 \%)$. Acetylation of 3 with acetic anhydride in pyridine $(\rightarrow 4)$, followed by cleavage of acetonide in 90\% TFA $(\rightarrow \mathbf{5}, 92 \%)$ and regioselective condensation with 2,3,4,6-tetra- $O$-benzoyl- $\alpha$-D-galactopyranose trichloroacetimidate (6) furnished trisaccharide 7. Deacylation of 7 in ammonia-saturated methanol furnished the methyl glycoside of sanqi oligosaccharide repeating unit 8 in $61 \%$ yield (from $\mathbf{5}$, Scheme 1).

To further study structure-activity relationships, we turned our attention to the synthesis of sanqi oligosaccharide analogues. Thus, 6-O-tert-butyldiphenylsilyl1,2- $O$-ethylidene- $\alpha$-D-galactopyranose ${ }^{8} \quad$ (9) was glycosylated with $\mathbf{6}$ in the presence of TMSOTf to give the $(1 \rightarrow 3)$-linked disaccharide $10(94 \%)$ which was subsequently desilylated with $\mathrm{TBAF}^{9}$ in THF to give the 4,6-diol 11. Coupling of $\mathbf{1 1}$ with donor $\mathbf{1}$ under standard glycosylation conditions gave trisaccharide $\mathbf{1 2}$ (54\%). Trisaccharide imidate 15 was prepared in 64\% 
overall yield via protection group manipulation of $\mathbf{1 2}$, i.e., de-ethylidenation, ${ }^{8}$ acetylation $(\rightarrow 13)$, deacetylation $^{10}$ on the anomeric carbon $(\rightarrow \mathbf{1 4})$ and finally Schmidt activation. ${ }^{11}$ Condensation of $\mathbf{1 5}$ and diosgenin furnished saponin derivative $\mathbf{1 6}$ that was treated with aqueous $1 \mathrm{~N} \mathrm{NaOH}$ in methanol $(\mathrm{pH}$ 9) to give the completed sanqi saponin analogue $17(60 \%$ for two steps).

With the aim to synthesize the dimerized sanqi oligosaccharide, disaccharide $\mathbf{5}$ was coupled with phenyl 2,6-di- $O$-benzoyl-3,4- $O$-isopropylidene-1-thio- $\beta$ D-galactopyranoside ${ }^{7}(\mathbf{1 8})$ to yield methyl 2,6-di- $O$-benzoyl-3,4- $O$-isopropylidene- $\beta$-D-galactopyranosyl- $(1 \rightarrow 3)$ [2,3,5-tri- $O$-benzoyl- $\alpha$-L-arabinofuranosyl- $(1 \rightarrow 6)]-2-O$ acetyl- $\alpha$-D-galactopyranoside (19), which was then acetylated in pyridine with acetic anhydride to give $\mathbf{2 0}$. Removal of the acetonide group in 90\% TFA then afforded the trisaccharide 3,4-diol 21 in a total yield of $42 \%$ from 5 (Scheme 2).

The coupling reaction of $\mathbf{1 5}$ and $\mathbf{2 1}$ was carried out in anhydrous $\mathrm{CH}_{2} \mathrm{Cl}_{2}$ in the presence of TMSOTf at $0{ }^{\circ} \mathrm{C}$. Surprisingly, a $41 \%$ yield of the $\alpha$ - $(1 \rightarrow 4)$-linked dimer 22 was isolated from the reaction mixture. To determine the correct assignments of this intriguing structure, we have run ${ }^{1} \mathrm{H}$ NMR, coupled ${ }^{13} \mathrm{C}$ NMR and ${ }^{1} \mathrm{H}-{ }^{1} \mathrm{H},{ }^{1} \mathrm{H}-{ }^{13} \mathrm{C}$ COSY experiments. The $\mathrm{H}-1$ of sugar residue III appears at $\delta 5.21 \mathrm{ppm}\left(J_{1,2} 3.6 \mathrm{~Hz}\right)$ in the ${ }^{1} \mathrm{H}$ NMR, while the corresponding $\mathrm{C}-1^{\mathrm{III}}$ at $\delta 98.5 \mathrm{ppm}$ $\left(J_{\mathrm{C}-1, \mathrm{H}-1} 171 \mathrm{~Hz}\right)$ in ${ }^{13} \mathrm{C}$ NMR spectroscopy, indicating an $\alpha$ linkage between carbohydrate units II and III in 22. Compared to acceptor 21, the chemical shift of C-4 ${ }^{\mathrm{II}}$ in 22 moved downfield to $\delta 78.6 \mathrm{ppm}$ from $\delta 68.8$ ppm, while the C-3 $3^{\mathrm{II}} \mathrm{S}$ of $\mathbf{2 1}$ and $\mathbf{2 2}$ appear at $\delta 72.2$ and $\delta 71.9 \mathrm{ppm}$ in ${ }^{13} \mathrm{C}$ NMR spectra, respectively, which confirms the C-4 glycosylation of unit II. Furthermore, acetylated 22 gave $\mathrm{H}-3^{\mathrm{II}}$ at $\delta 5.08$ ppm, further confirming this assignment. Decreasing the reaction temperature $\left(-40^{\circ} \mathrm{C}\right.$ ) and/or adding more TMSOTf (up to 0.4 equiv) did not improve the yield of this coupling reaction. The major byproducts showed ${ }^{1} \mathrm{H}$ NMR spectra that could not be identified and gave smaller masses compared to that of 22. Full deprotection of $\mathbf{2 2}$ in ammonia-saturated methanol gave the $\alpha-(1 \rightarrow 4)$-linked sanqi dimer $\mathbf{2 3}$ in $93 \%$ yield. The potential bioactivity of compounds $\mathbf{8}, \mathbf{1 7}$ and $\mathbf{2 3}$ is currently under investigation.

\section{Experimental}

General methods.-Optical rotations were determined at $25^{\circ} \mathrm{C}$ with a Perkin-Elmer model $241 \mathrm{MC}$

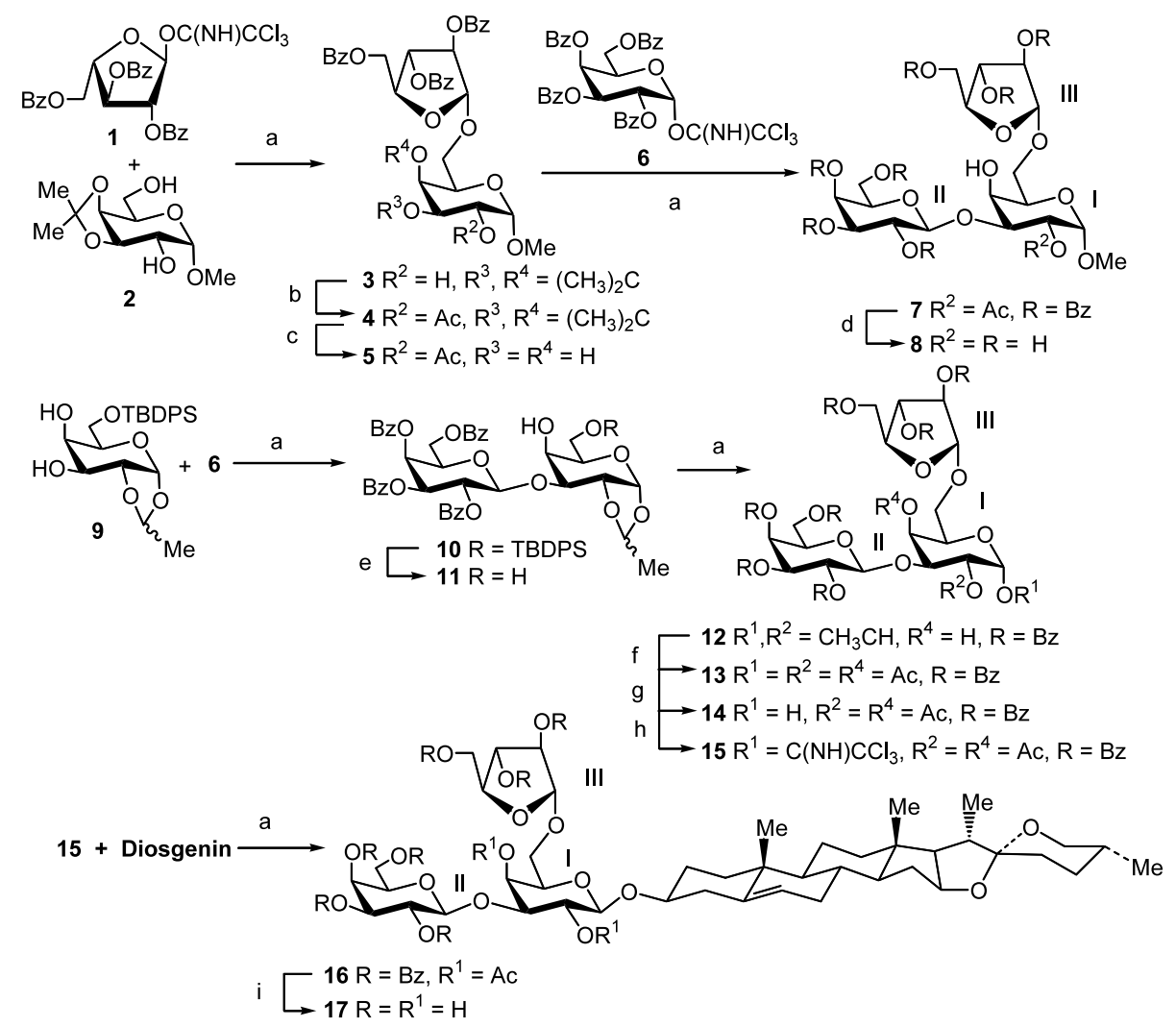

Scheme 1. Reagents and conditions: (a) TMSOTf, $\mathrm{CH}_{2} \mathrm{Cl}_{2} ; 76 \%$ for 3; $82 \%$ for 7; 94\% for 10; 61\% for 16; (b) Ac $\mathrm{O}$, Pyr; (c) $90 \%$ TFA; 92\%; (d) $\mathrm{NH}_{3}, \mathrm{MeOH} ; 74 \%$; (e) TBAF, THF; 77\%; (f) 90\% TFA; Ac $2 \mathrm{O}, \mathrm{Pyr} ;$ (g) $\mathrm{NH}_{3}, \mathrm{THF}^{-\mathrm{MeOH}}$ (7:3); 69\% from 12; (h) $\mathrm{Cl}_{3} \mathrm{CCN}, \mathrm{DBU} ; 93 \%$; (i) $1 \mathrm{~N} \mathrm{NaOH}, \mathrm{MeOH} ; 99 \%$. 


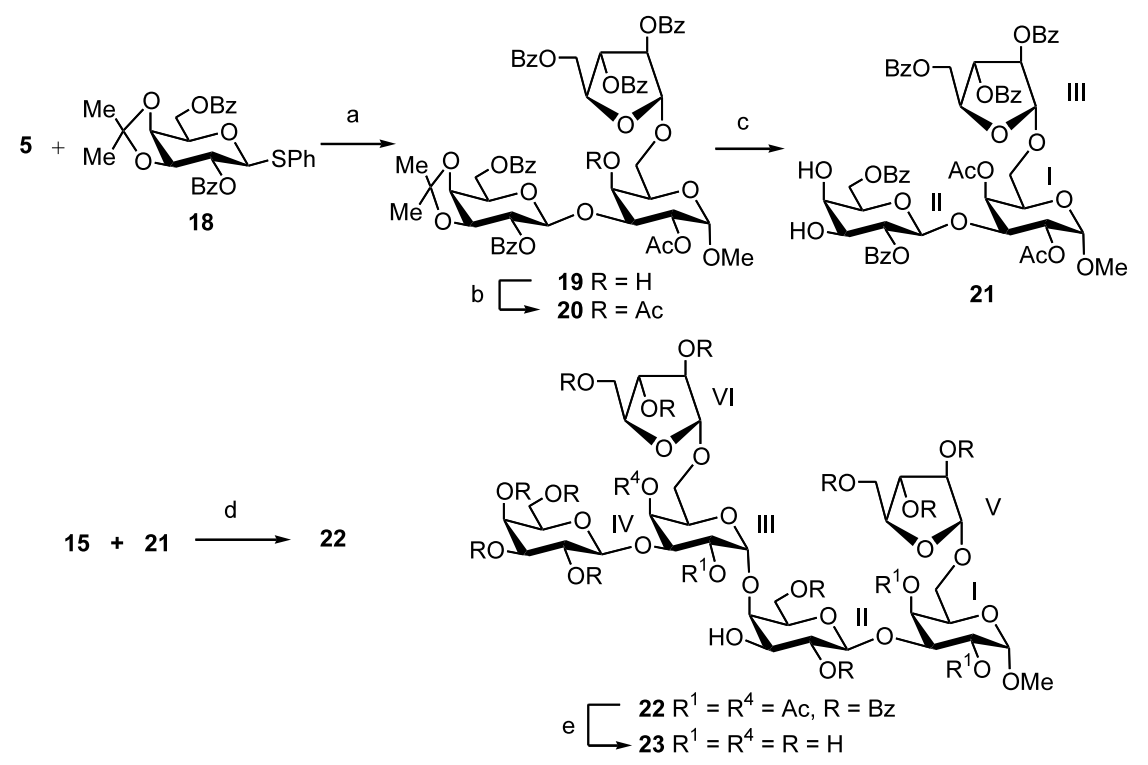

Scheme 2. (a) NIS, TMSOTf; 45\%; (b) $\mathrm{Ac}_{2} \mathrm{O}$, Pyr; (c) $90 \%$ TFA; 94\%; (d) TMSOTf, $\mathrm{CH}_{2} \mathrm{Cl}_{2} ; 41 \%$; (e) $\mathrm{NH}_{3}, \mathrm{MeOH}^{2}$; $\%$.

automatic polarimeter. ${ }^{1} \mathrm{H}$ NMR, ${ }^{13} \mathrm{C}$ NMR and ${ }^{1} \mathrm{H}-$ ${ }^{1} \mathrm{H},{ }^{1} \mathrm{H}-{ }^{13} \mathrm{C}$ COSY spectra were recorded with ARX 400 spectrometers for solutions in $\mathrm{CDCl}_{3}, \mathrm{CD}_{3} \mathrm{OD}$ and $\mathrm{D}_{2} \mathrm{O}$. Chemical shifts are given in ppm downfield from internal $\mathrm{Me}_{4} \mathrm{Si}$. Mass spectra were measured using MALDITOF-MS with $\alpha$-cyano-4-hydroxycinnamic acid (CCA) as the matrix, or recorded with a $\mathrm{VG}$ PLATFORM mass spectrometer using the electrospray ionization (ESI) technique to introduce the sample. High-resolution thin-layer chromatography (HRTLC) was performed on Silica Gel $\mathrm{HF}_{254}$ with detection by charring with $30 \%(\mathrm{v} / \mathrm{v}) \mathrm{H}_{2} \mathrm{SO}_{4}$ in $\mathrm{MeOH}$ or in some cases by UV detection. Column chromatography was conducted by elution of a column $(16 \times 240 \mathrm{~mm}, 18 \times$ $300 \mathrm{~mm}, 35 \times 400 \mathrm{~mm})$ of silica gel $(100-200$ mesh) with EtOAc-petroleum ether (bp $60-90^{\circ} \mathrm{C}$ ) as the eluent. Solutions were concentrated at $<60^{\circ} \mathrm{C}$ under diminished pressure.

Methyl 2,3,5-tri-O-benzoyl- $\alpha$-L-arabinofuranosyl$(1 \rightarrow 6)$-2-O-acetyl-3,4-O-isopropylidene- $\alpha$-D-galactopyranoside (4).- To a mixture of 1 (11.16 g, 18.4 $\mathrm{mmol})$ and $2(3.52 \mathrm{~g}, 15 \mathrm{mmol})$ in dry $\mathrm{CH}_{2} \mathrm{Cl}_{2}(100 \mathrm{~mL})$ at $0{ }^{\circ} \mathrm{C}$ was added TMSOTf $(85 \mu \mathrm{L}, 0.47 \mathrm{mmol})$. The mixture was stirred at this temperature for $2.5 \mathrm{~h}$, then neutralized with $\mathrm{Et}_{3} \mathrm{~N}$ and concentrated. The residue was subjected to column chromatography on silica gel with 2:1 petroleum ether-EtOAc as the eluent to give $\mathbf{3}$ $(7.7 \mathrm{~g}, 76 \%)$. To a mixture of $3(7.15 \mathrm{~g}, 10.5 \mathrm{mmol})$ in pyridine $(15 \mathrm{~mL})$ was added $\mathrm{Ac}_{2} \mathrm{O}(2.5 \mathrm{~mL})$. The mixture was stirred at $\mathrm{rt}$ for $10 \mathrm{~h}$, then co-evaporated with toluene to dryness. The residue was subjected to silica gel column chromatography with $3: 1$ petroleum etherEtOAc as the eluent to give 4 as a syrup (6.7 g, 88\%): $[\alpha]_{\mathrm{D}}+78^{\circ}\left(c 1, \mathrm{CHCl}_{3}\right) ;{ }^{1} \mathrm{H}$ NMR $\left(400 \mathrm{MHz}, \mathrm{CDCl}_{3}\right)$ : $1.24,1.52\left(2 \mathrm{~s}, 6 \mathrm{H}, 2 \mathrm{CH}_{3}\right), 2.13\left(\mathrm{~s}, 3 \mathrm{H}, \mathrm{COCH}_{3}\right), 3.38$ $\left(\mathrm{s}, 3 \mathrm{H}, \mathrm{OCH}_{3}\right), 3.85\left(\mathrm{dd}, 1 \mathrm{H}, J_{6 \mathrm{a}, 6 \mathrm{~b}} 10.3, J_{6 \mathrm{a}, 5} 7.1 \mathrm{~Hz}\right.$, H-6a), 4.10 (dd, $\left.1 \mathrm{H}, J_{6 \mathrm{~b}, 5} 5.4 \mathrm{~Hz}, \mathrm{H}-6 \mathrm{~b}\right), 4.26$ (ddd, 1 $\left.\mathrm{H}, J_{5,4} 2.4 \mathrm{~Hz}, \mathrm{H}-5\right), 4.27\left(\mathrm{dd}, 1 \mathrm{H}, J_{4,3} 5.3 \mathrm{~Hz}, \mathrm{H}-4\right)$, $4.31\left(\mathrm{dd}, 1 \mathrm{H}, J_{3,2} 8.0 \mathrm{~Hz}, \mathrm{H}-3\right), 4.60-4.62(\mathrm{~m}, 1 \mathrm{H}$, $\left.\mathrm{H}-4^{\prime}\right), 4.70\left(\mathrm{dd}, 1 \mathrm{H}, J_{5 \mathrm{a}^{\prime}, 5 \mathrm{~b}^{\prime}} 11.9, J_{5 \mathrm{a}^{\prime}, 4^{\prime}} 4.9 \mathrm{~Hz}, \mathrm{H}-5 \mathrm{a}^{\prime}\right)$, $4.84\left(\mathrm{~d}, 1 \mathrm{H}, J_{1,2} 3.6 \mathrm{~Hz}, \mathrm{H}-1\right), 4.87$ (dd, $1 \mathrm{H}, J_{5 \mathrm{~b}^{\prime}, 4^{\prime}} 3.4$ Hz, H-5b'), 4.97 (dd, 1 H, H-2), 5.41 (s, $1 \mathrm{H}, \mathrm{H}-\mathrm{l}^{\prime}$ ), 5.56 $\left(\mathrm{d}, 1 \mathrm{H}, J_{3^{\prime}, 4^{\prime}} 5.0 \mathrm{~Hz}, \mathrm{H}-3^{\prime}\right), 5.63\left(\mathrm{~s}, 1 \mathrm{H}, \mathrm{H}-2^{\prime}\right), 7.27-$ $8.06(\mathrm{~m}, 15 \mathrm{H}, \mathrm{Ph})$; Anal. Calcd for $\mathrm{C}_{38} \mathrm{H}_{40} \mathrm{O}_{14}: \mathrm{C}$, 63.33; H, 5.55. Found: C, 63.60; H, 5.48.

Methyl 2,3,5-tri-O-benzoyl- $\alpha$-L-arabinofuranosyl$(1 \rightarrow 6)$-2-O-acetyl- $\alpha$-D-galactopyranoside (5). - A solution of $4(5 \mathrm{~g}, 6.93 \mathrm{mmol})$ in $\mathrm{CH}_{2} \mathrm{Cl}_{2}(2 \mathrm{~mL})$ and $90 \%$ TFA was stirred at $\mathrm{rt}$ for about $10 \mathrm{~min}$ at which time TLC indicated the reaction was complete. The mixture was neutralized with aq $\mathrm{NaHCO}_{3}$, then extracted with $\mathrm{CH}_{2} \mathrm{Cl}_{2}$. The organic phase was dried over $\mathrm{Na}_{2} \mathrm{SO}_{4}$ and concentrated. The residue was subjected to column chromatography on silica gel with 2:1 petroleum etherEtOAc as the eluent to give 5 as a syrup (4.34 g, 92\%): $[\alpha]_{\mathrm{D}}+52^{\circ}\left(c 1, \mathrm{CHCl}_{3}\right) ;{ }^{1} \mathrm{H}$ NMR $\left(300 \mathrm{MHz}, \mathrm{CDCl}_{3}\right)$ : $2.06\left(\mathrm{~s}, 3 \mathrm{H}, \mathrm{COCH}_{3}\right), 3.29\left(\mathrm{~s}, 3 \mathrm{H}, \mathrm{OCH}_{3}\right), 3.75-3.78$ (m, 1 H, H-6a), 3.91-4.01 (m, 3 H, H-3, H-5 and H-6b), 4.04 (d, $\left.1 \mathrm{H}, J_{4,3} 3.3 \mathrm{~Hz}, \mathrm{H}-4\right), 4.58-4.63$ (m, 2 $\mathrm{H}, \mathrm{H}-4^{\prime}$ and $\left.\mathrm{H}-5 \mathrm{a}^{\prime}\right), 4.70-4.80$ (m, 1 H, H-5b'), 4.82 (d, $\left.1 \mathrm{H}, J_{1,2} 3.6 \mathrm{~Hz}, \mathrm{H}-1\right), 4.96$ (dd, $1 \mathrm{H}, J_{2,3} 10.2 \mathrm{~Hz}, \mathrm{H}-2$ ), 5.30 (s, $\left.1 \mathrm{H}, \mathrm{H}-2^{\prime}\right), 5.46$ (s, $\left.1 \mathrm{H}, \mathrm{H}-1^{\prime}\right), 5.51(\mathrm{~d}, 1 \mathrm{H}, J$ $\left.3.0 \mathrm{~Hz}, \mathrm{H}-3^{\prime}\right), 7.19-8.00(\mathrm{~m}, 15 \mathrm{H}, \mathrm{Ph})$; Anal. Calcd for $\mathrm{C}_{35} \mathrm{H}_{36} \mathrm{O}_{14}: \mathrm{C}, 61.76 ; \mathrm{H}, 5.33$. Found: $\mathrm{C}, 61.55 ; \mathrm{H}, 5.42$. Methyl 2,3,5-tri-O-benzoyl- $\alpha$-L-arabinofuranosyl$(1 \rightarrow 6)$-[2,3,4,6-tetra-O-benzoyl- $\beta$-D-galactopyranosyl$(1 \rightarrow 3)]-2-\mathrm{O}-$ acetyl- $\alpha$-D-galactopyranoside (7). - To a solution of $5(0.8 \mathrm{~g}, 1.17 \mathrm{mmol})$ and $6(1.023 \mathrm{~g}, 1.38$ mmol) in dry $\mathrm{CH}_{2} \mathrm{Cl}_{2}(5 \mathrm{~mL})$ at $0{ }^{\circ} \mathrm{C}$ was added TMS- 
OTf $(23 \mu \mathrm{L}, 0.13 \mathrm{mmol})$. The mixture was stirred at this temperature for $2 \mathrm{~h}$, then neutralized with $\mathrm{Et}_{3} \mathrm{~N}$, and the solvents were evaporated under reduced pressure. The residue was subjected to column chromatography on silica gel with $1.5: 1$ to $1: 1$ petroleum ether-EtOAc as the eluent to give 7 as a foam $(1.215 \mathrm{~g}, 82 \%)$ : $[\alpha]_{\mathrm{D}}$ $+95^{\circ}\left(c 1, \mathrm{CHCl}_{3}\right) ;{ }^{1} \mathrm{H}$ NMR $\left(400 \mathrm{MHz}, \mathrm{CDCl}_{3}\right): 1.53$ $\left(\mathrm{s}, 3 \mathrm{H}, \mathrm{COCH}_{3}\right), 3.32\left(\mathrm{~s}, 3 \mathrm{H}, \mathrm{OCH}_{3}\right), 3.46(\mathrm{dd}, 1 \mathrm{H}$, $\left.J_{6 \mathrm{a}, 6 \mathrm{~b}} 11.2, J_{6 \mathrm{a}, 5} 3.1 \mathrm{~Hz}, \mathrm{H}-6 \mathrm{a}^{\mathrm{I}}\right), 3.90\left(\mathrm{dd}, 1 \mathrm{H}, J_{6 \mathrm{~b}, 5} 8.0\right.$ $\left.\mathrm{Hz}, \mathrm{H}-6 \mathrm{~b}^{\mathrm{I}}\right), 3.98\left(\mathrm{dd}, 1 \mathrm{H}, \mathrm{H}-5^{\mathrm{I}}\right), 4.10\left(\mathrm{dd}, 1 \mathrm{H}, J_{3,2}\right.$ $\left.10.2, J_{3,4} 3.2 \mathrm{~Hz}, \mathrm{H}-3^{\mathrm{I}}\right), 4.22\left(\mathrm{~d}, 1 \mathrm{H}, \mathrm{H}-4^{\mathrm{I}}\right), 4.32$ (br t, $\left.1 \mathrm{H}, \mathrm{H}-5^{\mathrm{II}}\right), 4.46\left(\mathrm{dd}, 1 \mathrm{H}, J_{6 \mathrm{a}, 6 \mathrm{~b}} 11.0, J_{6 \mathrm{a}, 5} 5.3 \mathrm{~Hz}\right.$, H-6a $\left.{ }^{\mathrm{II}}\right), 4.57-4.61$ (m, $2 \mathrm{H}, \mathrm{H}-6 \mathrm{~b}^{\mathrm{II}}$ and H-4 $\left.4^{\mathrm{III}}\right), 4.72(\mathrm{dd}$, $\left.1 \mathrm{H}, J_{5 \mathrm{a}, 5 \mathrm{~b}} 12.1, J_{5 \mathrm{a}, 4} 4.8 \mathrm{~Hz}, \mathrm{H}-5 \mathrm{a}^{\mathrm{III}}\right), 4.86(\mathrm{dd}, 1 \mathrm{H}$, $\left.J_{5 \mathrm{~b}, 4} 3.7 \mathrm{~Hz} \mathrm{H}-5 \mathrm{~b}^{\mathrm{III}}\right), 4.90\left(\mathrm{~d}, 1 \mathrm{H}, J_{1,2} 3.7 \mathrm{~Hz}, \mathrm{H}-1^{\mathrm{I}}\right)$, $5.00\left(\mathrm{~d}, 1 \mathrm{H}, J_{1,2} 8.0 \mathrm{~Hz}, \mathrm{H}-1^{\mathrm{II}}\right), 5.15\left(\mathrm{dd}, 1 \mathrm{H}, \mathrm{H}-2^{\mathrm{I}}\right)$, $5.38\left(\mathrm{~s}, 1 \mathrm{H}, \mathrm{H}-2^{\mathrm{III}}\right), 5.57\left(\mathrm{~s}, 1 \mathrm{H}, \mathrm{H}-1^{\mathrm{III}}\right), 5.59(\mathrm{~d}, 1 \mathrm{H}$, $\left.J_{3,4} 3.0 \mathrm{~Hz}, \mathrm{H}-3^{\mathrm{III}}\right), 5.62\left(\mathrm{dd}, 1 \mathrm{H}, J_{3,2} 10.4, J_{3,4} 3.4 \mathrm{~Hz}\right.$, $\left.\mathrm{H}-3^{\mathrm{II}}\right), 5.83\left(\mathrm{dd}, 1 \mathrm{H}, \mathrm{H}-2^{\mathrm{II}}\right), 5.98\left(\mathrm{~d}, 1 \mathrm{H}, \mathrm{H}-4^{\mathrm{II}}\right)$, 7.21-8.08 (m, $35 \mathrm{H}, \mathrm{Ph}) ;{ }^{13} \mathrm{C}$ NMR (100 MHz, $\left.\mathrm{CDCl}_{3}\right)$ : $20.00\left(\mathrm{COCH}_{3}\right), 54.96\left(\mathrm{OCH}_{3}\right), 62.01\left(\mathrm{C}-6^{\mathrm{II}}\right), 63.70$ $\left(\mathrm{C}-5^{\mathrm{III}}\right), 67.04\left(\mathrm{C}-6^{\mathrm{I}}\right), 67.93\left(\mathrm{C}-4^{\mathrm{II}}\right), 68.63\left(\mathrm{C}-2^{\mathrm{II}}\right), 69.11$ $\left(\mathrm{C}-2^{\mathrm{I}}\right), 69.31\left(\mathrm{C}-3^{\mathrm{II}}\right), 69.35\left(\mathrm{C}-5^{\mathrm{II}}\right), 71.33\left(\mathrm{C}-5^{\mathrm{I}}\right), 71.68$ $\left(\mathrm{C}-4^{\mathrm{I}}\right), 77.79\left(\mathrm{C}-3^{\mathrm{I}}\right), 77.94\left(\mathrm{C}-3^{\mathrm{III}}\right), 81.19\left(\mathrm{C}-4^{\mathrm{III}}\right), 81.91$ $\left(\mathrm{C}-2^{\mathrm{III}}\right), 96.76\left(\mathrm{C}-1^{\mathrm{I}}\right), 101.90\left(\mathrm{C}-1^{\mathrm{II}}\right), 106.05\left(\mathrm{C}-1^{\mathrm{III}}\right)$, $128.24,128.37,128.43,128.46,128.63,128.66,129.76$, $129.78,129.94,132.99,133.34,133.36,133.45,133.52$, $164.87,165.26,165.44,165.51,165.61,165.82,166.14$, 169.92; Anal. Calcd for $\mathrm{C}_{69} \mathrm{H}_{62} \mathrm{O}_{23}$ : C, 65.81; H, 4.96. Found: C, 65.60; H, 5.09.

Methyl $\alpha$-L-arabinofuranosyl- $(1 \rightarrow 6)-[\beta$-D-galactopyranosyl-( $1 \rightarrow 3)]-\alpha$-D-galactopyranoside $(\mathbf{8})$. - A solution of $7(0.7 \mathrm{~g}, 0.556 \mathrm{mmol})$ in ammonia-saturated $\mathrm{MeOH}$ $(100 \mathrm{~mL})$ was stirred at $\mathrm{rt}$ for 7 days. The solvents were evaporated, and the residue was purified on a Sephadex LH-20 column with $\mathrm{MeOH}$ as the eluent to give 8 as solid $(0.2 \mathrm{~g}, 74 \%):[\alpha]_{\mathrm{D}}+23^{\circ}\left(c 1, \mathrm{CH}_{3} \mathrm{OH}\right) ;{ }^{1} \mathrm{H} \mathrm{NMR}$ (400 MHz, $\left.\mathrm{CD}_{3} \mathrm{OD}\right): 3.41\left(\mathrm{~s}, 3 \mathrm{H}, \mathrm{OCH}_{3}\right), 3.56(\mathrm{~m}, 2$ $\mathrm{H}), 3.66-3.79(\mathrm{~m}, 6 \mathrm{H}), 3.88-3.93(\mathrm{~m}, 4 \mathrm{H}), 3.99-4.05$ $(\mathrm{m}, 4 \mathrm{H}), 4.22\left(\mathrm{~d}, 1 \mathrm{H}, J_{4,3} 2.4 \mathrm{~Hz}, \mathrm{H}-4^{\mathrm{I}}\right), 4.52(\mathrm{~d}, 1 \mathrm{H}$, $\left.J_{1,2} 7.6 \mathrm{~Hz}, \mathrm{H}-1^{\mathrm{II}}\right), 4.77\left(\mathrm{~d}, 1 \mathrm{H}, J_{1,2} 3.8 \mathrm{~Hz}, \mathrm{H}-1^{\mathrm{I}}\right), 5.00$ (s, $\left.1 \mathrm{H}, \mathrm{H}-1{ }^{\mathrm{III}}\right) ;{ }^{13} \mathrm{C}$ NMR $\left(100 \mathrm{MHz}, \mathrm{CD}_{3} \mathrm{OD}\right): 55.61$ $\left(\mathrm{OCH}_{3}\right), 62.46,62.88,68.22,70.00,70.13,70.52,70.62$, $72.88,74.45,76.52,78.65,81.52,83.40,85.43,100.99$, 106.34, 109.72; ESIMS: Calcd for $\mathrm{C}_{18} \mathrm{H}_{32} \mathrm{O}_{15}, 488.17$ [M]; Found, $487[\mathrm{M}-\mathrm{H}]^{+}$.

$2,3,4,6$-Tetra-O-benzoyl- $\beta$-D-galactopyranosyl-( $1 \rightarrow 3)$ 6-O-tert-butyldiphenylsilyl-1,2-O-ethylidene- $\alpha$-D-galactopyranose (10). - To a solution of $9(5.3 \mathrm{~g}, 11.9 \mathrm{mmol})$ and $6(9.34 \mathrm{~g}, 12.6 \mathrm{mmol})$ in $\mathrm{CH}_{2} \mathrm{Cl}_{2}(40 \mathrm{~mL})$ at $0{ }^{\circ} \mathrm{C}$ was added TMSOTf $(90 \mu \mathrm{L}, 0.5 \mathrm{mmol})$ under an $\mathrm{N}_{2}$ atmosphere. The mixture was stirred at this temperature for $1.5 \mathrm{~h}$ at which time TLC indicated the reaction was complete. It was then neutralized with $\mathrm{Et}_{3} \mathrm{~N}$ and concentrated. The residue was subjected to column chromatography on silica gel with 4:1 petroleum ether-
EtOAc as the eluent to give $\mathbf{1 0}(\mathrm{R}, \mathrm{S}$ mixture) as a syrup (11.46 g, 94\%): ${ }^{1} \mathrm{H}$ NMR $\left(300 \mathrm{MHz}, \mathrm{CDCl}_{3}\right)$ : $1.04\left(\mathrm{~s}, 9 \mathrm{H}, \mathrm{C}\left(\mathrm{CH}_{3}\right)_{3}\right), 1.24,1.40\left(2 \mathrm{~d}, 3 \mathrm{H}, \mathrm{CHCH}_{3}\right.$, the ratio of the isomers is about 1.7:1.3), 3.76-3.94 (m, $4 \mathrm{H}$ ), 4.09-4.15 (m, $1 \mathrm{H}, \mathrm{H}-5$ ), 4.32-4.47 (m, $3 \mathrm{H}, \mathrm{H}-4$, $\mathrm{H}-5^{\prime}$ and H-6a'), 4.56-4.64 (m, $\left.1 \mathrm{H}, \mathrm{H}-6 \mathrm{~b}^{\prime}\right), 5.20-5.26$ (m, $2 \mathrm{H}), 5.45$ (br d, $1 \mathrm{H}, \mathrm{H}-1), 5.60-5.80(\mathrm{~m}, 2 \mathrm{H})$, $6.00\left(\mathrm{~d}, 1 \mathrm{H}, J_{4^{\prime}, 3^{\prime}} 3 \mathrm{~Hz}, \mathrm{H}-4^{\prime}\right), 7.20-8.12(\mathrm{~m}, 30 \mathrm{H}, \mathrm{Ph})$; Anal. Calcd for $\mathrm{C}_{58} \mathrm{H}_{58} \mathrm{O}_{15} \mathrm{Si}$ : C, 68.09; H, 5.71. Found: C, 68.37; H, 5.69.

2,3,4,6-Tetra-O-benzoyl- $\beta$-D-galactopyranosyl-( $1 \rightarrow 3)$ 1,2-O-ethylidene- $\alpha$-D-galactopyranose (11). - To a solution of 10 (10.5 g, $10.3 \mathrm{mmol})$ in THF $(100 \mathrm{~mL})$ was added TBAF (7.048 g, $23.7 \mathrm{mmol})$. The mixture was stirred at $\mathrm{rt}$ for $2.5 \mathrm{~h}$ at which time TLC indicated the reaction was complete. The solvents were evaporated, and the residue was subjected to column chromatography on silica gel with 1:1 petroleum ether-EtOAc as the eluent to give $\mathbf{1 1}(6.2 \mathrm{~g}, 77 \%)$ as a syrup; MALDITOF-MS: Calcd for $\mathrm{C}_{42} \mathrm{H}_{40} \mathrm{O}_{15}, 784.24$ [M]; Found, $807.30[\mathrm{M}+\mathrm{Na}]^{+}$. Treatment of $11(20 \mathrm{mg})$ with $\mathrm{Ac}_{2} \mathrm{O}$ in pyridine gave 2,3,4,6-tetra- $O$-benzoyl- $\beta$ D-galactopyranosyl-( $1 \rightarrow 3)-4,6$-di- $O$-acetyl-1,2- $O$-ethylidene $-\alpha-\mathrm{D}$-galactopyranose as a syrup: ${ }^{1} \mathrm{H}$ NMR (400 $\mathrm{MHz}, \mathrm{CDCl}_{3}$ ): $1.23\left(\mathrm{~d}, 1.7 \mathrm{H}, J 3.6 \mathrm{~Hz}, \mathrm{CHCH}_{3}\right), 1.34$ (d, $\left.1.3 \mathrm{H}, J 3.6 \mathrm{~Hz}, \mathrm{CHCH}_{3}\right), 1.85\left(\mathrm{~s}, 1.7 \mathrm{H}, \mathrm{COCH}_{3}\right)$, $1.89\left(\mathrm{~s}, 1.3 \mathrm{H}, \mathrm{COCH}_{3}\right), 2.05,2.06$ (br s, $3 \mathrm{H}, \mathrm{COCH}_{3}$ ), $3.90\left(\mathrm{t}, 0.43 \mathrm{H}, J_{3,4} 4.0 \mathrm{~Hz}, \mathrm{H}-3\right), 4.06-4.19(\mathrm{~m}, 4 \mathrm{H})$, $4.24(\mathrm{t}, 0.57 \mathrm{H}), 4.34(\mathrm{t}, 1 \mathrm{H}), 4.40-4.47(\mathrm{~m}, 1 \mathrm{H})$, $4.63-4.69(\mathrm{~m}, 1 \mathrm{H}), 5.11\left(\mathrm{q}, 0.43 \mathrm{H}, J 4.8 \mathrm{~Hz}, \mathrm{CHCH}_{3}\right)$, $5.22-5.30(\mathrm{~m}, 1.57 \mathrm{H}), 5.39\left(\mathrm{~d}, 0.43 \mathrm{H}, J_{1^{\prime}, 2^{\prime}} 4.8 \mathrm{~Hz}\right)$, $5.49(\mathrm{~d}, 1 \mathrm{H}), 5.54$ (br s, $0.57 \mathrm{H}), 5.62-5.65($ br d, $1 \mathrm{H})$, 5.71-5.77 (m, 1 H), 5.99 (br s, $\left.1 \mathrm{H}, \mathrm{H}-4^{\prime}\right), 7.24-8.12$ $(\mathrm{m}, 20 \mathrm{H}, \mathrm{Ph})$.

2,3,5-Tri-O - benzoyl - $\alpha$ - L - arabinofuranosyl - $(1 \rightarrow 6)$ [2,3,4,6-tetra-O-benzoyl- $\beta$-D-galactopyranosyl- $(1 \rightarrow 3)]$ 1,2-O-ethylidene- $\alpha$-D-galactopyranose (12). - To a solution of 11 (2.4 g, $3.06 \mathrm{mmol})$ and $\mathbf{1}(1.89 \mathrm{~g}, 3.11 \mathrm{mmol})$ in $\mathrm{CH}_{2} \mathrm{Cl}_{2}(20 \mathrm{~mL})$ at $0{ }^{\circ} \mathrm{C}$ were added TMSOTf $(40$ $\mu \mathrm{L}, 0.22 \mathrm{mmol})$. The mixture was stirred at this temperature for $1 \mathrm{~h}$ at which time TLC indicated the reaction was complete. It was then neutralized with $\mathrm{Et}_{3} \mathrm{~N}$ and concentrated. The residue was subjected to column chromatography on silica gel with 2:1 petroleum etherEtOAc as the eluent to give 12 as a syrup (2.03 g, 54\%). One isomer gave $\left.{ }^{1} \mathrm{H} \mathrm{NMR} \mathrm{(300} \mathrm{MHz}, \mathrm{CDCl}_{3}\right)$ as follows: 1.17 (d, $\left.3 \mathrm{H}, J 4.8 \mathrm{~Hz}, \mathrm{CHCH}_{3}\right), 1.18(\mathrm{~s}, 3 \mathrm{H}$, $\left.\mathrm{COCH}_{3}\right), 3.59\left(\mathrm{dd}, 1 \mathrm{H}, J_{6 \mathrm{a}, 6 \mathrm{~b}} 10.2, J_{6 \mathrm{a}, 5} 6.3 \mathrm{~Hz}, \mathrm{H}-6 \mathrm{a}^{\mathrm{I}}\right)$, $3.79\left(\mathrm{dd}, 1 \mathrm{H}, J_{6 \mathrm{~b}, 5} 5.4 \mathrm{~Hz} \mathrm{H}-6 \mathrm{~b}^{\mathrm{I}}\right), 4.07-4.14(\mathrm{~m}, 3 \mathrm{H})$, $4.26(\mathrm{~m}, 1 \mathrm{H}), 4.41(\mathrm{dd}, 1 \mathrm{H}, J 11.4,6.0 \mathrm{~Hz}), 4.57-4.64$ $(\mathrm{m}, 3 \mathrm{H}), 4.79(\mathrm{~m}, 1 \mathrm{H}), 5.20-5.26(\mathrm{~m}, 3 \mathrm{H}), 5.48-$ $5.63(\mathrm{~m}, 5 \mathrm{H}), 5.75(\mathrm{dd}, 1 \mathrm{H}, J 10.5,7.6 \mathrm{~Hz}), 5.96(\mathrm{~d}$, $\left.1 \mathrm{H}, J_{4,3} 3.3 \mathrm{~Hz}, \mathrm{H}-4^{\mathrm{II}}\right), 7.23-8.12(\mathrm{~m}, 35 \mathrm{H}, \mathrm{Ph})$; MALDITOF-MS: Calcd for $\mathrm{C}_{68} \mathrm{H}_{60} \mathrm{O}_{22}, 1228.36$ [M]; Found, $1251[\mathrm{M}+\mathrm{Na}]^{+}$.

2,3,5-Tri-O - benzoyl - $\alpha$ - L - arabinofuranosyl - $(1 \rightarrow 6)$ [2,3,4,6-tetra-O-benzoyl- $\beta$-D-galactopyranosyl- $(1 \rightarrow 3)]$ - 
2,4-di-O-acetyl- $\alpha$-D-galactopyranosyl trichloroacetimidate (15). - To a solution of $\mathbf{1 2}(1.52 \mathrm{~g}, 1.24 \mathrm{mmol})$ in $\mathrm{CH}_{2} \mathrm{Cl}_{2}(2 \mathrm{~mL})$ was added aq $90 \%$ TFA $(15 \mathrm{~mL})$. The mixture was stirred at $\mathrm{rt}$ for $2 \mathrm{~h}$, then co-evaporated with toluene under reduced pressure. The residue was dissolved in pyridine $(10 \mathrm{~mL})$ and $\mathrm{Ac}_{2} \mathrm{O}(5 \mathrm{~mL})$, and stirred at $\mathrm{rt}$ for $6 \mathrm{~h}$, then concentrated to dryness to give crude 13. Crude $\mathbf{1 3}$ dissolved in 7:3 ammonia-saturated $\mathrm{THF}-\mathrm{MeOH}(100 \mathrm{~mL})$ was stirred at $\mathrm{rt}$ for 30 min, and the solvents were then evaporated at $35^{\circ} \mathrm{C}$. The residue was subjected to column chromatography on silica gel with 1.5:1 petroleum ether-EtOAc as the eluent to give $\mathbf{1 4}$ as a syrup $(1.1 \mathrm{~g}, 69 \%$ from 12). Compound $14(0.73 \mathrm{~g}, 0.567 \mathrm{mmol})$ was dissolved in $\mathrm{CH}_{2} \mathrm{Cl}_{2}(6 \mathrm{~mL})$, then $\mathrm{CCl}_{3} \mathrm{CN}(0.5 \mathrm{~mL}, 0.5 \mathrm{mmol})$ and DBU $(50 \mu \mathrm{L})$ were added at $0{ }^{\circ} \mathrm{C}$. The mixture was stirred at $\mathrm{rt}$ for $2 \mathrm{~h}$, then concentrated. The residue was subjected to column chromatography on silica gel with 1.5:1 petroleum ether-EtOAc as eluent to give $\mathbf{1 5}$ as a syrup $(0.749 \mathrm{~g}, 93 \%):[\alpha]_{\mathrm{D}}+59^{\circ}\left(\mathrm{c} 1, \mathrm{CHCl}_{3}\right) ;{ }^{1} \mathrm{H}$ NMR (400 MHz, $\left.\mathrm{CDCl}_{3}\right): 1.51\left(\mathrm{~s}, 3 \mathrm{H}, \mathrm{COCH}_{3}\right), 2.23$ (s, $\left.3 \mathrm{H}, \mathrm{COCH}_{3}\right), 3.68\left(\mathrm{dd}, 1 \mathrm{H}, J_{6 \mathrm{a}, 6 \mathrm{~b}} 11.0, J_{6 \mathrm{a}, 5} 4.3 \mathrm{~Hz}\right.$, H-6a $\left.{ }^{\mathrm{I}}\right), 3.82\left(\mathrm{dd}, 1 \mathrm{H}, J_{6 \mathrm{~b}, 5} 4.8 \mathrm{~Hz}, \mathrm{H}-6 \mathrm{~b}^{\mathrm{I}}\right), 4.18(\mathrm{dd}, 1$ $\left.\mathrm{H}, \mathrm{H}-5^{\mathrm{II}}\right), 4.27\left(\mathrm{dd}, 1 \mathrm{H}, J_{3,2} 10.2, J_{3,4} 3.2 \mathrm{~Hz}, \mathrm{H}-3^{\mathrm{I}}\right)$, 4.29-4.36 (m, 2 H, H-5 $5^{\mathrm{I}}$ and $\left.\mathrm{H}-6 \mathrm{a}^{\mathrm{II}}\right), 4.59-4.69(\mathrm{~m}, 3$ $\mathrm{H}, \mathrm{H}-6 \mathrm{~b}^{\mathrm{II}}, \mathrm{H}-4^{\mathrm{III}}$ and $\left.\mathrm{H}-5 \mathrm{a}^{\mathrm{III}}\right), 4.85\left(\mathrm{dd}, 1 \mathrm{H}, J_{5 \mathrm{~b}, 5 \mathrm{a}} 11.9\right.$, $\left.J_{5 \mathrm{~b}, 4} 3.1 \mathrm{~Hz}, \mathrm{H}-5 \mathrm{~b}^{\mathrm{III}}\right), 4.97\left(\mathrm{~d}, 1 \mathrm{H}, J_{1,2} 7.8 \mathrm{~Hz}, \mathrm{H}-1^{\mathrm{II}}\right)$, $5.23\left(\mathrm{dd}, 1 \mathrm{H}, J_{2,1} 3.6 \mathrm{~Hz}, \mathrm{H}-2^{\mathrm{I}}\right), 5.53-5.58(\mathrm{~m}, 3 \mathrm{H}$, $\mathrm{H}-3^{\mathrm{II}}, \mathrm{H}-3^{\mathrm{III}}$ and $\left.\mathrm{H}-2^{\mathrm{III}}\right), 5.72\left(\mathrm{dd}, 1 \mathrm{H}, J_{2,3} 10.4 \mathrm{~Hz}\right.$, $\left.\mathrm{H}-2^{\mathrm{II}}\right), 5.83\left(\mathrm{~d}, 1 \mathrm{H}, \mathrm{H}-4^{\mathrm{I}}\right), 5.91\left(\mathrm{~d}, 1 \mathrm{H}, J_{4,3} 3.2 \mathrm{~Hz}\right.$, $\left.\mathrm{H}-4^{\mathrm{II}}\right), 6.46\left(\mathrm{~d}, 1 \mathrm{H}, \mathrm{H}-1^{\mathrm{I}}\right), 7.25-8.11(\mathrm{~m}, 35 \mathrm{H}, \mathrm{Ph})$, 8.49 (s, $1 \mathrm{H}, \mathrm{NH}) ;{ }^{13} \mathrm{C}$ NMR $\left(100 \mathrm{MHz}, \mathrm{CDCl}_{3}\right): 19.72$ $\left(\mathrm{COCH}_{3}\right), 20.72\left(\mathrm{COCH}_{3}\right), 61.63\left(\mathrm{C}-6^{\mathrm{II}}\right), 63.52\left(\mathrm{C}-5^{\mathrm{III}}\right)$, $65.82\left(\mathrm{C}-6^{\mathrm{I}}\right), 67.66\left(\mathrm{C}-4^{\mathrm{II}}\right), 68.64\left(\mathrm{C}-2^{\mathrm{I}}\right), 69.26\left(\mathrm{C}-4^{\mathrm{I}}\right)$, $69.93\left(\mathrm{C}-2^{\mathrm{II}}\right), 71.17\left(\mathrm{C}-5^{\mathrm{II}}\right), 71.31\left(\mathrm{C}-5^{\mathrm{I}}\right), 71.31\left(\mathrm{C}-3^{\mathrm{II}}\right)$, $73.84\left(\mathrm{C}-3^{\mathrm{I}}\right), 77.95\left(\mathrm{C}-3^{\mathrm{III}}\right), 81.16\left(\mathrm{C}-4^{\mathrm{III}}\right), 82.12\left(\mathrm{C}-2^{\mathrm{III}}\right)$, $93.56\left(\mathrm{C}-1^{\mathrm{I}}\right), 101.16\left(\mathrm{C}-1^{\mathrm{II}}\right), 106.03\left(\mathrm{C}-1^{\mathrm{III}}\right), 128.17$, $128.25,128.43,128.48,128.61,128.95,128.99,129.12$, $129.18,129.31,129.57,129.69,129.72,129.81,129.88$, $130.06,132.97,133.32,133.46,133.61,160.53,164.69$, $165.26,165.39,165.51,165.74,165.85,166.16,169.64$, 169.73; MALDITOF-MS: Calcd for $\mathrm{C}_{72} \mathrm{H}_{62} \mathrm{Cl}_{3} \mathrm{NO}_{24}$, 1429.27 [M]; Found, $1452.17[\mathrm{M}+\mathrm{Na}]^{+}$. Anal. Calcd for $\mathrm{C}_{72} \mathrm{H}_{62} \mathrm{Cl}_{3} \mathrm{NO}_{24}: \mathrm{C}, 60.41 ; \mathrm{H}, 4.37$. Found: $\mathrm{C}, 60.68$; $\mathrm{H}, 4.29$.

Diosgenyl 2,3,4,6-tetra-O-benzoyl- $\beta$-D-galactopyranosyl-(1 $\rightarrow 3)$-[2,3,5-tri-O-benzoyl- $\alpha$-L-arabinofuranosyl$(1 \rightarrow 6)]-2,4-d i$-O-acetyl- $\beta$-D-galactopyranoside (16).To a solution of $15(0.53 \mathrm{~g}, 0.37 \mathrm{mmol})$ and diosgenin $(0.185 \mathrm{~g}, 0.45 \mathrm{mmol})$ in $\mathrm{CH}_{2} \mathrm{Cl}_{2}(2 \mathrm{~mL})$ at $0{ }^{\circ} \mathrm{C}$ was added TMSOTf $(25 \mu \mathrm{L}, 0.14 \mathrm{mmol})$. The mixture was stirred at this temperature for about $1 \mathrm{~h}$, then neutralized with $\mathrm{Et}_{3} \mathrm{~N}$ and concentrated. The residue was subjected to column chromatography on silica gel with 1.5:1 petroleum ether-EtOAc as the eluent to give $\mathbf{1 6}$ $(0.38 \mathrm{~g}, 61 \%)$ as a foam: $[\alpha]_{\mathrm{D}}+30^{\circ}\left(c 1, \mathrm{CHCl}_{3}\right) ;{ }^{1} \mathrm{H}$
NMR (400 MHz, $\left.\mathrm{CDCl}_{3}\right): 0.71\left(\mathrm{~s}, 3 \mathrm{H}, \mathrm{CH}_{3}\right), 0.79$ (d, $\left.3 \mathrm{H}, J 6.4 \mathrm{~Hz}, \mathrm{CH}_{3}\right), 0.81\left(\mathrm{~s}, 3 \mathrm{H}, \mathrm{CH}_{3}\right), 0.84-0.87$ (m, $2 \mathrm{H}), 0.97\left(\mathrm{~d}, 3 \mathrm{H}, J 6.8 \mathrm{~Hz}, \mathrm{CH}_{3}\right), 1.01-1.15(\mathrm{~m}, 2 \mathrm{H})$, $1.21-1.30(\mathrm{~m}, 5 \mathrm{H}), 1.42-2.09(\mathrm{~m}, 24 \mathrm{H}), 1.55(\mathrm{~s}, 3 \mathrm{H}$, $\left.\mathrm{COCH}_{3}\right), 2.17\left(\mathrm{~s}, 3 \mathrm{H}, \mathrm{COCH}_{3}\right), 3.34(\mathrm{~m}, 1 \mathrm{H}, 3 \alpha-\mathrm{H})$, 3.37 (t, $1 \mathrm{H}, J 10.9 \mathrm{~Hz}, \mathrm{H}-26 \mathrm{a}), 3.47$ (dd, $1 \mathrm{H}, J_{26 \mathrm{~b}, 25} 4.1$ $\mathrm{Hz}, \mathrm{H}-26 \mathrm{~b}), 3.72$ (dd, $1 \mathrm{H}, J_{6 \mathrm{a}, 6 \mathrm{~b}} 12.1, J_{6 \mathrm{a}, 5} 8.4 \mathrm{~Hz}$, H-6a ${ }^{\mathrm{I}}$ ), 3.79-3.83 (m $2 \mathrm{H}, \mathrm{H}-5^{\mathrm{I}}$ and H-6b ${ }^{\mathrm{I}}$ ), 3.87 (dd, 1 $\left.\mathrm{H}, J_{3,2} 10.0, J_{3,4} 3.6 \mathrm{~Hz}, \mathrm{H}-3^{\mathrm{I}}\right), 4.17\left(\mathrm{t}, 1 \mathrm{H}, \mathrm{H}-5^{\mathrm{II}}\right), 4.29$ $\left(\mathrm{dd}, J_{6 \mathrm{a}, 6 \mathrm{~b}} 11.2, J_{6 \mathrm{a}, 5} 7.0 \mathrm{~Hz}, \mathrm{H}-6 \mathrm{a}^{\mathrm{II}}\right), 4.38\left(\mathrm{~d}, 1 \mathrm{H}, J_{1,2}\right.$ $\left.8.1 \mathrm{~Hz}, \mathrm{H}-1^{\mathrm{I}}\right), 4.29(\mathrm{t}, 1 \mathrm{H}, J 6.8 \mathrm{~Hz}, \mathrm{H}-16), 4.58(\mathrm{~m}, 3$ $\mathrm{H}, \mathrm{H}-6 \mathrm{a}^{\mathrm{II}}, \mathrm{H}-4^{\mathrm{III}}$ and $\left.\mathrm{H}-5 \mathrm{a}^{\mathrm{III}}\right), 4.82\left(\mathrm{dd}, 1 \mathrm{H}, J_{5 \mathrm{~b}, 5 \mathrm{a}} 11.9\right.$, $\left.J_{5 \mathrm{~b}, 4} 3.3 \mathrm{~Hz}, \mathrm{H}-5 \mathrm{~b}^{\mathrm{III}}\right), 4.88$ (d, $\left.1 \mathrm{H}, J_{1,2} 8.0 \mathrm{~Hz}, \mathrm{H}-1^{\mathrm{II}}\right)$, $5.11\left(\mathrm{dd}, 1 \mathrm{H}, \mathrm{H}-2^{\mathrm{I}}\right), 5.24\left(\mathrm{~d}, J_{6,7 \mathrm{a}} 4 \mathrm{~Hz}, \mathrm{H}-6\right.$ of diosgenyl), 5.34 (s, $\left.1 \mathrm{H}, \mathrm{H}-1^{\mathrm{III}}\right), 5.51-5.56(\mathrm{~m}, 3 \mathrm{H}$, $\mathrm{H}-3^{\mathrm{II}}, \mathrm{H}-3^{\mathrm{III}}$ and $\left.\mathrm{H}-2^{\mathrm{III}}\right), 5.60\left(\mathrm{~d}, 1 \mathrm{H}, \mathrm{H}-4^{\mathrm{I}}\right), 5.69(\mathrm{dd}$, $\left.1 \mathrm{H}, J_{2,3} 10.4 \mathrm{~Hz}, \mathrm{H}-2^{\mathrm{II}}\right), 5.90$ (d, $\left.1 \mathrm{H}, J_{4,3} 4 \mathrm{~Hz}, \mathrm{H}-4^{\mathrm{II}}\right)$, 7.23-8.09 (m, 35 H, Ph); ${ }^{13} \mathrm{C}$ NMR (100 MHz, $\left.\mathrm{CDCl}_{3}\right)$ : 14.57 (C-21), 16.25 (C-18), 17.17 (C-27), 19.15 (C-19), $20.22\left(\mathrm{COCH}_{3}\right), 20.68(\mathrm{C}-11), 20.85\left(\mathrm{COCH}_{3}\right), 26.93$ (C-24), 29.44 (C-2), 30.31 (C-25), 31.32 (C-23), 31.40 (C-8), 31.84 (C-15), 31.98 (C-7), 36.69 (C-10), 36.84 (C-1), 38.95 (C-4), 39.71 (C-12), 40.23 (C-13), 41.61 (C-20), 49.75 (C-9), 56.38 (C-14), $61.65\left(\mathrm{C}-6^{\mathrm{II}}\right), 62.12$ (C-17), 63.68 (C-5 $\left.{ }^{\mathrm{III}}\right), 66.60\left(\mathrm{C}-6^{\mathrm{I}}\right), 66.86(\mathrm{C}-26), 67.73$ $\left(\mathrm{C}-4^{\mathrm{II}}\right), 69.36\left(\mathrm{C}-4^{\mathrm{I}}\right), 69.93\left(\mathrm{C}-2^{\mathrm{II}}\right), 70.62\left(\mathrm{C}-2^{\mathrm{I}}\right), 71.19$ $\left(\mathrm{C}-5^{\mathrm{II}}\right), 71.47\left(\mathrm{C}-3^{\mathrm{II}}\right), 73.22\left(\mathrm{C}-5^{\mathrm{I}}\right), 77.76\left(\mathrm{C}-3^{\mathrm{I}}\right), 77.92$ (C-3 $\left.{ }^{\text {III }}\right), 80.10$ (C-3), 80.81 (C-16), 80.92 (C-4 $\left.{ }^{\text {III }}\right), 82.23$ $\left(\mathrm{C}-2^{\mathrm{III}}\right), 100.40\left(\mathrm{C}-1^{\mathrm{I}}\right), 101.43\left(\mathrm{C}-1^{\mathrm{II}}\right), 106.38\left(\mathrm{C}-1^{\mathrm{III}}\right)$, 109.29 (C-22), 121.553 (C-6), 128.24, 128.33, 128.51, $128.54,128.68,129.02,129.19,129.38,129.45,129.77$, $129.88,129.95,130.10,133.05,133.24,133.34,133.55$, 133.66, 140.45 (C-5), 164.74, 165.32, 165.46, 165.61, $165.73,165.91,166.17,168.66,170.11$; MALDITOFMS: Calcd for $\mathrm{C}_{97} \mathrm{H}_{102} \mathrm{O}_{26}, 1682.67$ [M]; Found, 1705.31 $[\mathrm{M}+\mathrm{Na}]^{+}$. Anal. Calcd for $\mathrm{C}_{97} \mathrm{H}_{102} \mathrm{O}_{26}: \mathrm{C}$, 69.19; H, 6.11. Found: C, 69.51; H, 5.93.

Diosgenyl $\beta$-D-galactopyranosyl- $(1 \rightarrow 3)$ - $[\alpha$-L-arabinofuranosyl- $(1 \rightarrow 6)]-\beta$-D-galactopyranoside $(\mathbf{1 7})$. - To a solution of $16(0.32 \mathrm{~g}, 0.19 \mathrm{mmol})$ in $\mathrm{MeOH}(100 \mathrm{~mL})$ was added aq $1 \mathrm{~N} \mathrm{NaOH}$ until $\mathrm{pH} 9-10$ was attained. The mixture was stirred at $\mathrm{rt}$ overnight, then neutralized with Amberlite IR-120 $\left(\mathrm{H}^{+}\right)$. The solvents were evaporated, and the residue was subjected to column chromatography on Sephadex LH-20 with $\mathrm{MeOH}$ as the eluent to give $\mathbf{1 7}$ as an amorphous solid $(0.16 \mathrm{~g}$, 99\%): $[\alpha]_{\mathrm{D}}-51^{\circ}\left(c 1, \mathrm{CH}_{3} \mathrm{OH}\right) ;{ }^{1} \mathrm{H}$ NMR $(400 \mathrm{MHz}$, $\mathrm{CD}_{3} \mathrm{OD}$ ): $0.81\left(\mathrm{~d}, 3 \mathrm{H}, J 6.4 \mathrm{~Hz}, \mathrm{CH}_{3}\right), 0.83$ (s, $3 \mathrm{H}$, $\left.\mathrm{CH}_{3}\right), 0.98\left(\mathrm{~d}, 3 \mathrm{H}, \mathrm{J} 6.8 \mathrm{~Hz}, \mathrm{CH}_{3}\right), 1.07\left(\mathrm{~s}, 3 \mathrm{H}, \mathrm{CH}_{3}\right)$, 0.97-1.0 (m, 1 H), 1.09-2.04 (m, $25 \mathrm{H}), 2.29(\mathrm{t}, 1 \mathrm{H}, J$ $12.0 \mathrm{~Hz}), 2.45(\mathrm{~d}, 1 \mathrm{H}, J 10.8 \mathrm{~Hz}), 3.32-4.13(\mathrm{~m}, 27 \mathrm{H})$, $4.41(\mathrm{t}, 1 \mathrm{H}, J 7.6 \mathrm{~Hz}, \mathrm{H}-16), 4.42\left(\mathrm{~d}, 1 \mathrm{H}, J_{1,2} 7.0 \mathrm{~Hz}\right.$, $\left.\mathrm{H}-1^{\mathrm{I}}\right), 4.44\left(\mathrm{~d}, 1 \mathrm{H}, J_{1,2} 7.6 \mathrm{~Hz}, \mathrm{H}-1^{\mathrm{II}}\right), 4.95(\mathrm{~s}, 1 \mathrm{H}$, $\mathrm{H}-1^{\mathrm{III}}$ ), 5.41 (br s, $1 \mathrm{H}, \mathrm{H}-6$ of diosgenyl); ${ }^{13} \mathrm{C}$ NMR (100 MHz, DOCD 3 ): 14.90 (C-21), 16.79 (C-18), 17.50 (C-27), 19.87 (C-19), 21.99 (C-11), 29.88 (C-24), 30.76 
(C-25), 31.43 (C-23), 32.42 (C-15), 32.74 (C-7), 32.79 (C-2), 33.17 (C-8), 37.99 (C-10), 38.48 (C-1), 39.75 (C-12), 40.92 (C-13), 41.41 (C-20), 42.89 (C-4), 51.61

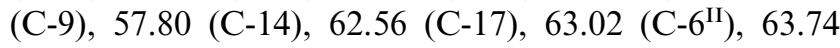
$\left(\mathrm{C}-5^{\mathrm{III}}\right), 67.84(\mathrm{C}-26), 67.96\left(\mathrm{C}-6^{\mathrm{I}}\right), 69.85\left(\mathrm{C}-4^{\mathrm{II}}\right), 70.24$ $\left(\mathrm{C}-4^{\mathrm{I}}\right), 71.51\left(\mathrm{C}-2^{\mathrm{II}}\right), 71.56\left(\mathrm{C}-2^{\mathrm{I}}\right), 74.60\left(\mathrm{C}-5^{\mathrm{II}}\right), 74.63$ $\left(\mathrm{C}-3^{\mathrm{II}}\right), 76.72\left(\mathrm{C}-5^{\mathrm{I}}\right), 78.88(\mathrm{C}-3), 80.04(\mathrm{C}-16), 82.19$ $\left(\mathrm{C}-3^{\mathrm{I}}\right), 83.43\left(\mathrm{C}-3^{\mathrm{III}}\right), 84.73\left(\mathrm{C}-4^{\mathrm{III}}\right), 85.70 \quad\left(\mathrm{C}-2^{\mathrm{III}}\right)$, $102.63\left(\mathrm{C}-1^{\mathrm{I}}\right), 106.27\left(\mathrm{C}-1^{\mathrm{II}}\right), 109.29(\mathrm{C}-22), 110.55(\mathrm{C}-$ $\left.1^{\mathrm{III}}\right), 122.50$ (C-6), 142.04 (C-5); MALDITOF-MS: Calcd for $\mathrm{C}_{44} \mathrm{H}_{70} \mathrm{O}_{17}, 870.46[\mathrm{M}]$; Found $893.39[\mathrm{M}+$ $\mathrm{Na}]^{+}$.

Methyl 2,3,5-tri-O-benzoyl- $\alpha$-L-arabinofuranosyl$(1 \rightarrow 6)$ - [2,6-di-O-benzoyl-3,4-O-isopropylidene - $\beta$ - Dgalactopyranosyl- $(1 \rightarrow 3)]-2-\mathrm{O}$-acetyl- $\alpha$-D-galactopyranoside (19). - To a mixture of $5(1.22 \mathrm{~g}, 1.76 \mathrm{mmol})$ and $18(1.2 \mathrm{~g}, 2.3 \mathrm{mmol})$ in dry $\mathrm{CH}_{2} \mathrm{Cl}_{2}(10 \mathrm{~mL})$ at $-20{ }^{\circ} \mathrm{C}$ were added $N$-iodosuccinimide (NIS, $0.8 \mathrm{~g}, 3.57 \mathrm{mmol}$ ) and TMSOTf $(22 \mu \mathrm{L}, 0.12 \mathrm{mmol})$. The mixture was stirred at this temperature for $80 \mathrm{~min}$, at which time TLC indicated the reaction was complete. The mixture was neutralized with $\mathrm{Et}_{3} \mathrm{~N}$, and concentrated. The residue was subjected to column chromatography on silica gel with 1.5:1 petroleum ether-EtOAc as the eluent to give syrupy $19(0.87 \mathrm{~g}, 45 \%):[\alpha]_{\mathrm{D}}+28^{\circ}(\mathrm{c} 1$, $\left.\mathrm{CHCl}_{3}\right) ;{ }^{1} \mathrm{H}$ NMR (400 MHz, $\left.\mathrm{CDCl}_{3}\right): 1.26,1.58$ (s, 6 $\left.\mathrm{H}, 2 \mathrm{CH}_{3}\right), 1.64\left(\mathrm{~s}, 3 \mathrm{H}, \mathrm{COCH}_{3}\right), 3.28\left(\mathrm{~s}, 3 \mathrm{H}, \mathrm{OCH}_{3}\right)$, $3.64\left(\mathrm{~d}, 1 \mathrm{H}, J 9.2 \mathrm{~Hz}, \mathrm{H}-6 \mathrm{a}^{\mathrm{I}}\right), 3.83-3.88\left(\mathrm{~m}, 2 \mathrm{H}, \mathrm{H}-4^{\mathrm{I}}\right.$, $\left.\mathrm{H}-6 \mathrm{~b}^{\mathrm{I}}\right), 3.96\left(\mathrm{~d}, 1 \mathrm{H}, J 9.6 \mathrm{~Hz}, \mathrm{H}-5^{\mathrm{I}}\right), 4.26\left(\mathrm{~s}, 1 \mathrm{H}, J_{4,3}\right.$ $4.1 \mathrm{~Hz}, \mathrm{H}-4^{\mathrm{II}}$ ), 4.26 (br s, $\left.1 \mathrm{H}, \mathrm{H}-3^{\mathrm{I}}\right), 4.32$ (br s, $1 \mathrm{H}$, $\left.\mathrm{H}-3^{\mathrm{II}}\right), 4.40$ (br s, $\left.1 \mathrm{H}, \mathrm{H}-5^{\mathrm{II}}\right), 4.54\left(\mathrm{~d}, 1 \mathrm{H}, \mathrm{H}-4^{\mathrm{III}}\right)$, $4.58-4.73$ (m, 4 H, H-5a $\left.{ }^{\mathrm{III}}, \mathrm{H}-5 \mathrm{~b}^{\mathrm{III}}, \mathrm{H}-1^{\mathrm{II}}, \mathrm{H}-6 \mathrm{a}^{\mathrm{II}}\right), 4.81$ $\left(\mathrm{d}, 1 \mathrm{H}, J 10.8 \mathrm{~Hz}, \mathrm{H}-6 \mathrm{~b}^{\mathrm{II}}\right), 4.85$ (d, $1 \mathrm{H}, J_{1,2} 4.4 \mathrm{~Hz}$, H-1 ${ }^{\mathrm{I}}$ ), 5.07 (dd, $1 \mathrm{H}, J_{2,3} 6.8 \mathrm{~Hz}, \mathrm{H}-2^{\mathrm{I}}$ ), 5.25 (br s, $1 \mathrm{H}$, $\left.\mathrm{H}-2^{\mathrm{II}}\right), 5.45\left(\mathrm{~s}, 1 \mathrm{H}, \mathrm{H}-1^{\mathrm{III}}\right), 5.53\left(\mathrm{~s}, 1 \mathrm{H}, \mathrm{H}-2^{\mathrm{III}}\right), 5.55$ (d, $\left.1 \mathrm{H}, J_{3,4} 4.0 \mathrm{~Hz}, \mathrm{H}-3^{\mathrm{III}}\right), 7.27-8.04$; MALDITOF-MS: Calcd for $\mathrm{C}_{58} \mathrm{H}_{58} \mathrm{O}_{21}, 1090.35$ [M]; Found, $1113[\mathrm{M}+$ $\mathrm{Na}]^{+}$. Anal. Calcd for $\mathrm{C}_{58} \mathrm{H}_{58} \mathrm{O}_{21}: \mathrm{C}, 63.85 ; \mathrm{H}, 5.36$. Found: C, 64.04; H, 5.19.

Methyl 2,3,5-tri-O-benzoyl- $\alpha$-L-arabinofuranosyl$(1 \rightarrow 6)$ - [2,6-di-O-benzoyl-3,4-O-isopropylidene - $\beta$ - Dgalactopyranosyl- $(1 \rightarrow 3)]-2,4-d i-\mathrm{O}$-acetyl- $\alpha$-D-galactopyranoside (20). - Compound 19 (1.85 g, $1.69 \mathrm{mmol})$ and $\mathrm{Ac}_{2} \mathrm{O}(1 \mathrm{~mL})$ were dissolved in pyridine $(5 \mathrm{~mL})$ at rt. The mixture was stirred at $\mathrm{rt}$ for $10 \mathrm{~h}$, then co-evaporated with toluene. The residue was subjected to column chromatography on silica gel with $3: 1$ petroleum ether-EtOAc as the eluent to give quantitative 20 as a syrup: $[\alpha]_{\mathrm{D}}+11^{\circ}\left(c 1, \mathrm{CHCl}_{3}\right) ;{ }^{1} \mathrm{H}$ NMR $\left(400 \mathrm{MHz}, \mathrm{CDCl}_{3}\right): 1.35,1.61\left(2 \mathrm{~s}, 6 \mathrm{H}, \mathrm{CH}_{3}\right), 1.73$, $2.10\left(2 \mathrm{~s}, 6 \mathrm{H}, \mathrm{COCH}_{3}\right), 3.27\left(\mathrm{~s}, 3 \mathrm{H}, \mathrm{OCH}_{3}\right), 3.56(\mathrm{dd}$, $\left.1 \mathrm{H}, J_{6 \mathrm{a}, 6 \mathrm{~b}} 10.9, J_{6 \mathrm{a}, 5} 3.2 \mathrm{~Hz}, \mathrm{H}-6 \mathrm{a}^{\mathrm{I}}\right), 3.91\left(\mathrm{dd}, 1 \mathrm{H}, J_{6 \mathrm{~b}, 5}\right.$ $\left.4.0 \mathrm{~Hz}, \mathrm{H}-6 \mathrm{~b}^{\mathrm{I}}\right), 4.00-4.03\left(\mathrm{~m}, 1 \mathrm{H}, \mathrm{H}-5^{\mathrm{I}}\right), 4.09-4.11(\mathrm{~m}$, $\left.1 \mathrm{H}, \mathrm{H}-4^{\mathrm{II}}\right), 4.13\left(\mathrm{dd}, 1 \mathrm{H}, J_{3,2} 10.2, J_{3,4} 3.4 \mathrm{~Hz}, \mathrm{H}-3^{\mathrm{I}}\right)$, $4.32\left(\mathrm{dd}, 1 \mathrm{H}, \mathrm{H}-3^{\mathrm{II}}\right), 4.38-4.42\left(\mathrm{~m}, 1 \mathrm{H}, \mathrm{H}-5^{\mathrm{II}}\right), 4.59-$ $4.62\left(\mathrm{~m}, 2 \mathrm{H}, \mathrm{H}-5 \mathrm{a}^{\mathrm{III}}\right.$ and $\left.\mathrm{H}-4^{\mathrm{III}}\right), 4.66-4.73(\mathrm{~m}, 3 \mathrm{H}$,
$\left.\mathrm{H}-5 \mathrm{~b}^{\mathrm{III}}, \mathrm{H}-1^{\mathrm{II}}, \mathrm{H}-6 \mathrm{a}^{\mathrm{II}}\right), 4.81\left(\mathrm{dd}, 1 \mathrm{H}, J_{6 \mathrm{~b}, 5} 3.2 \mathrm{~Hz}\right.$, $\left.\mathrm{H}-6 \mathrm{~b}^{\mathrm{II}}\right), 4.87$ (d, $\left.1 \mathrm{H}, J_{1,2} 3.6 \mathrm{~Hz}, \mathrm{H}-1^{\mathrm{I}}\right), 4.95(\mathrm{dd}, 1 \mathrm{H}$, $\left.\mathrm{H}-2^{\mathrm{I}}\right), 5.25\left(\mathrm{t}, 1 \mathrm{H}, J_{1,2} 7.3 \mathrm{~Hz}, \mathrm{H}-2^{\mathrm{II}}\right), 5.33(\mathrm{~s}, 1 \mathrm{H}$, $\left.\mathrm{H}-1^{\mathrm{III}}\right), 5.52\left(\mathrm{~s}, 1 \mathrm{H}, \mathrm{H}-2^{\mathrm{III}}\right), 5.53\left(\mathrm{~d}, 1 \mathrm{H}, \mathrm{H}-4^{\mathrm{I}}\right), 5.56(\mathrm{~d}$, $\left.1 \mathrm{H}, J_{3,4} 4.8 \mathrm{~Hz}, \mathrm{H}-3^{\mathrm{III}}\right), 7.26-8.10(\mathrm{~m}, 25 \mathrm{H}, \mathrm{Ph})$; MALDITOF-MS: Calcd for $\mathrm{C}_{60} \mathrm{H}_{60} \mathrm{O}_{22}, 1132.36$ [M]; Found, $1155.4[\mathrm{M}+\mathrm{Na}]^{+}$.

Methyl 2,3,5-tri-O-benzoyl- $\alpha$-L-arabinofuranosyl$(1 \rightarrow 6)$-[2,6-di-O-benzoyl- $\beta$-D-galactopyranosyl- $(1 \rightarrow 3)]$ 2,4-di-O-acetyl- $\alpha$-D-galactopyranoside $(\mathbf{2 1})$. $-\mathrm{A}$ solution of $20(1.00 \mathrm{~g}, 1.13 \mathrm{mmol})$ in $\mathrm{CH}_{2} \mathrm{Cl}_{2}(2 \mathrm{~mL})$ and $90 \%$ TFA were stirred at $\mathrm{rt}$ for about $10 \mathrm{~min}$, at which time TLC indicated the reaction was complete. The mixture was neutralized with aq $\mathrm{NaHCO}_{3}$, then extracted with $\mathrm{CH}_{2} \mathrm{Cl}_{2}$. The organic phase was dried over $\mathrm{Na}_{2} \mathrm{SO}_{4}$ and concentrated. The residue was subjected to column chromatography on silica gel with 1:1 petroleum ether-EtOAc as the eluent to give $\mathbf{2 1}$ as a syrup $(0.915 \mathrm{~g}, 94 \%):[\alpha]_{\mathrm{D}}+38^{\circ}\left(\mathrm{c} 1, \mathrm{CHCl}_{3}\right) ;{ }^{1} \mathrm{H}$ NMR $\left(400 \mathrm{MHz}, \mathrm{CDCl}_{3}\right): 1.68,2.11(2 \mathrm{~s}, 6 \mathrm{H}, 2$ $\left.\mathrm{COCH}_{3}\right), 3.28\left(\mathrm{~s}, 3 \mathrm{H}, \mathrm{OCH}_{3}\right), 3.60\left(\mathrm{dd}, 1 \mathrm{H}, J_{6 \mathrm{a}, 6 \mathrm{~b}} 10.9\right.$, $\left.J_{6 \mathrm{a}, 5} 3.1 \mathrm{~Hz}, \mathrm{H}-6 \mathrm{a}^{\mathrm{I}}\right), 3.76-3.84\left(\mathrm{~m}, 3 \mathrm{H}, \mathrm{H}-3^{\mathrm{II}}, \mathrm{H}-5^{\mathrm{II}}\right.$ and $\left.\mathrm{H}-6 \mathrm{~b}^{\mathrm{I}}\right), 4.01$ (br s, $1 \mathrm{H}, \mathrm{H}-5^{\mathrm{I}}$ and $\left.\mathrm{H}-4^{\mathrm{II}}\right), 4.16(\mathrm{dd}, 1 \mathrm{H}$, $\left.J_{3,2} 10.4, J_{3,4} 3.2 \mathrm{~Hz}, \mathrm{H}-3^{\mathrm{I}}\right), 4.58-4.62\left(\mathrm{~m}, 3 \mathrm{H}, \mathrm{H}-4^{\mathrm{III}}\right.$, $\mathrm{H}-5 \mathrm{a}^{\mathrm{III}}$ and $\left.\mathrm{H}-5 \mathrm{~b}^{\mathrm{III}}\right), 4.68\left(\mathrm{dd}, 1 \mathrm{H}, J_{6 \mathrm{a}, 6 \mathrm{~b}} 12.0, J_{6 \mathrm{a}, 5} 4.4\right.$ $\left.\mathrm{Hz}, \mathrm{H}-6 \mathrm{a}^{\mathrm{II}}\right), 4.69$ (d, $\left.1 \mathrm{H}, J_{1,2} 7.3 \mathrm{~Hz}, \mathrm{H}-1^{\mathrm{II}}\right), 4.83$ (dd, $\left.1 \mathrm{H}, J_{6 \mathrm{~b}, 5} 3.2 \mathrm{~Hz}, \mathrm{H}-6 \mathrm{~b}^{\mathrm{II}}\right), 4.87\left(4 \mathrm{H}, \mathrm{d}, J_{1,2} 3.6 \mathrm{~Hz}\right.$, $\left.\mathrm{H}-1^{\mathrm{I}}\right), 4.94\left(\mathrm{dd}, 1 \mathrm{H}, \mathrm{H}-2^{\mathrm{I}}\right), 5.18\left(\mathrm{dd}, 1 \mathrm{H}, J_{2,3} 9.6 \mathrm{~Hz}\right.$, $\left.\mathrm{H}-2^{\mathrm{II}}\right), 5.33$ (s, $\left.1 \mathrm{H}, \mathrm{H}-1^{\mathrm{III}}\right), 5.54$ (s, $\left.1 \mathrm{H}, \mathrm{H}-2^{\mathrm{III}}\right), 5.55$ (d, $\left.1 \mathrm{H}, \mathrm{H}-4^{\mathrm{I}}\right), 5.56\left(\mathrm{~d}, J_{3,4} 5.2 \mathrm{~Hz}, \mathrm{H}-3^{\mathrm{III}}\right), 7.26-8.08$ (m, $25 \mathrm{H}, \mathrm{Ph}) ;{ }^{13} \mathrm{C}$ NMR $\left(100 \mathrm{MHz}, \mathrm{CDCl}_{3}\right): 20.28,27.77$, $55.14,62.51\left(\mathrm{C}-5^{\mathrm{III}}\right), 63.55\left(\mathrm{C}-6^{\mathrm{II}}\right), 66.32\left(\mathrm{C}-6^{\mathrm{I}}\right), 68.41$ $\left(\mathrm{C}-5^{\mathrm{I}}\right), 68.77\left(\mathrm{C}-4^{\mathrm{II}}\right), 70.22\left(\mathrm{C}-2^{\mathrm{I}}\right), 70.80\left(\mathrm{C}-4^{\mathrm{I}}\right), 72.26$ $\left(\mathrm{C}-3^{\mathrm{II}}\right), 72.46\left(\mathrm{C}-5^{\mathrm{II}}\right), 72.60\left(\mathrm{C}-3^{\mathrm{I}}\right), 73.68\left(\mathrm{C}-2^{\mathrm{II}}\right), 77.85$ $\left(\mathrm{C}-3^{\mathrm{III}}\right), 81.00\left(\mathrm{C}-4^{\mathrm{III}}\right), 82.04\left(\mathrm{C}-2^{\mathrm{III}}\right), 96.75\left(\mathrm{C}-1^{\mathrm{I}}\right)$, $101.26\left(\mathrm{C}-1^{\mathrm{II}}\right), 106.17\left(\mathrm{C}-1^{\mathrm{III}}\right), 128.28,128.42,128.47$, $128.96,129.08,129.58,129.64,129.68,129.72,129.87$, $133.012133 .22,133.27,133.46,133.50,165.30$, 165.75, 166.17, 166.33, 169.83, 170.76; MALDITOF-MS: Calcd for $\mathrm{C}_{57} \mathrm{H}_{56} \mathrm{O}_{22}, 1092.33[\mathrm{M}]$; Found, $1115.17[\mathrm{M}+\mathrm{Na}]^{+}$ . Anal. Calcd for $\mathrm{C}_{57} \mathrm{H}_{56} \mathrm{O}_{22}$ : C, 62.63; H, 5.16. Found: C, 62.88; H, 5.02.

Methyl 2,3,4,6-tetra-O-benzoyl- $\beta$-D-galactopyranosyl-( $1 \rightarrow 3)$-[2,3,5-tri-O-benzoyl- $\alpha$-L-arabinofuranosyl$(1 \rightarrow 6)]-2-\mathrm{O}$-acetyl- $\alpha$-D-galactopyranosyl- $(1 \rightarrow 4)-2,6$ di-O-benzoyl- $\beta$ - D-galactopyranosyl- $(1 \rightarrow 3)$-[2,3,5-tri$\mathrm{O}$-benzoyl- $\alpha$ - L-arabinofuranosyl- $(1 \rightarrow 6)]-2,4-d i$-O-acetyl- $\alpha$-D-galactopyranoside (22). - To a solution of $\mathbf{1 5}$ $(0.794 \mathrm{~g}, 0.56 \mathrm{mmol})$ and $21(0.519 \mathrm{~g}, 0.47 \mathrm{mmol})$ in dry $\mathrm{CH}_{2} \mathrm{Cl}_{2}(5 \mathrm{~mL})$ at $0{ }^{\circ} \mathrm{C}$ was added TMSOTf $(10 \mu \mathrm{L}$, $0.06 \mathrm{mmol})$. The mixture was stirred at this temperature for $2 \mathrm{~h}$, then neutralized with $\mathrm{Et}_{3} \mathrm{~N}$, and the solvents were evaporated. The residue was subjected to column chromatography on silica gel with 1.5:1 petroleum ether-EtOAc as the eluent to give 22 as an amorphous solid $(0.46 \mathrm{~g}, 41 \%):[\alpha]_{\mathrm{D}}+91^{\circ}\left(c 1, \mathrm{CHCl}_{3}\right) ;{ }^{1} \mathrm{H}$ NMR 
(400 MHz, $\left.\mathrm{CDCl}_{3}\right): 1.51\left(\mathrm{~s}, 3 \mathrm{H}, \mathrm{COCH}_{3}\right), 1.60(\mathrm{~s}, 6 \mathrm{H}$, $\left.2 \mathrm{COCH}_{3}\right), 2.12\left(\mathrm{~s}, 3 \mathrm{H}, \mathrm{COCH}_{3}\right), 3.31\left(\mathrm{~s}, 3 \mathrm{H}, \mathrm{OCH}_{3}\right)$, $3.63-3.66\left(\mathrm{~m}, 2 \mathrm{H}, \mathrm{H}-6 \mathrm{a}^{\mathrm{I}}\right.$ and $\left.\mathrm{H}-6 \mathrm{a}^{\mathrm{III}}\right), 3.72(\mathrm{dd}, 1 \mathrm{H}$, $\left.J_{6 \mathrm{~b}, 6 \mathrm{a}} 11.3, J_{6 \mathrm{~b}, 5} 4.3 \mathrm{~Hz}, \mathrm{H}-6 \mathrm{~b}^{\mathrm{III}}\right), 3.83-3.86(\mathrm{~m}, 3 \mathrm{H}$, $\mathrm{H}-3^{\mathrm{II}}, \mathrm{H}-6 \mathrm{~b}^{\mathrm{I}}$ and $\left.\mathrm{H}-5^{\mathrm{II}}\right), 4.03\left(\mathrm{~d}, 1 \mathrm{H}, J_{4,3} 2.4 \mathrm{~Hz}, \mathrm{H}-4^{\mathrm{II}}\right)$, $4.10\left(\mathrm{~m}, 2 \mathrm{H}, \mathrm{H}-6 \mathrm{a}^{\mathrm{II}}\right.$ and $\left.\mathrm{H}-5^{\mathrm{I}}\right), 4.20$ (dd, $1 \mathrm{H}, J_{3,2} 10.4$, $\left.J_{3,4} 3.5 \mathrm{~Hz}, \mathrm{H}-3^{\mathrm{I}}\right), 4.25-4.35\left(\mathrm{~m}, 2 \mathrm{H}, \mathrm{H}-6 \mathrm{a}^{\mathrm{IV}}\right.$ and $\left.\mathrm{H}-5^{\mathrm{IV}}\right), 4.51-4.60\left(\mathrm{~m}, 5 \mathrm{H}, \mathrm{H}-3^{\mathrm{III}}, \mathrm{H}-4^{\mathrm{VI}}, \mathrm{H}-5 \mathrm{a}^{\mathrm{V}}, \mathrm{H}-4^{\mathrm{V}}\right.$ and $\left.\mathrm{H}-6 \mathrm{~b}^{\mathrm{II}}\right), 4.65-4.73\left(\mathrm{~m}, 4 \mathrm{H}, \mathrm{H}-6 \mathrm{~b}^{\mathrm{IV}}, \mathrm{H}-5 \mathrm{~b}^{\mathrm{V}}, \mathrm{H}-5 \mathrm{a}^{\mathrm{VI}}\right.$, $\left.\mathrm{H}-1^{\mathrm{II}}\right), 4.80\left(\mathrm{dd}, 1 \mathrm{H}, J_{5 \mathrm{~b}, 5 \mathrm{a}} 11.8, J_{5 \mathrm{~b}, 4} 3.2 \mathrm{~Hz}, \mathrm{H}-5 \mathrm{~b}^{\mathrm{VI}}\right)$, $4.85\left(\mathrm{~m}, 1 \mathrm{H}, \mathrm{H}-5^{\mathrm{III}}\right), 4.92\left(\mathrm{~d}, 1 \mathrm{H}, J_{1,2} 3.6 \mathrm{~Hz}, \mathrm{H}-1^{\mathrm{I}}\right)$, $5.03\left(\mathrm{dd}, 1 \mathrm{H}, \mathrm{H}-2^{\mathrm{I}}\right), 5.16\left(\mathrm{~d}, 1 \mathrm{H}, J_{1,2} 7.7 \mathrm{~Hz}, \mathrm{H}-1^{\mathrm{IV}}\right)$, $5.21\left(\mathrm{~d}, 1 \mathrm{H}, J_{1,2} 3.6 \mathrm{~Hz}, \mathrm{H}-1^{\mathrm{III}}\right), 5.22\left(\mathrm{dd}, 1 \mathrm{H}, J_{2,3} 10.4\right.$ $\left.\mathrm{Hz}, \mathrm{H}-2^{\mathrm{III}}\right), 5.32\left(\mathrm{dd}, 1 \mathrm{H}, J_{2,3} 10.4, J_{2,1} 7.6 \mathrm{~Hz}, \mathrm{H}-2^{\mathrm{II}}\right)$, $5.34\left(\mathrm{~s}, 1 \mathrm{H}, \mathrm{H}-1^{\mathrm{v}}\right), 5.36\left(\mathrm{~s}, 1 \mathrm{H}, \mathrm{H}-1^{\mathrm{VI}}\right), 5.42(\mathrm{~d}, 1 \mathrm{H}$, $\left.J_{2,3} 1.6 \mathrm{~Hz}, \mathrm{H}-2^{\mathrm{VI}}\right), 5.54-5.62\left(\mathrm{~m}, 4 \mathrm{H}, \mathrm{H}-2^{\mathrm{V}}, \mathrm{H}-3^{\mathrm{V}}\right.$, $\left.\mathrm{H}-4^{\mathrm{I}}, \mathrm{H}-3^{\mathrm{VI}}\right), 5.63\left(\mathrm{dd}, 1 \mathrm{H}, J_{3,2} 10.4, J_{3,4} 3.3 \mathrm{~Hz}\right.$, $\left.\mathrm{H}-3^{\mathrm{IV}}\right), 5.77\left(\mathrm{dd}, 1 \mathrm{H}, \mathrm{H}-2^{\mathrm{IV}}\right), 5.81\left(\mathrm{~d}, 1 \mathrm{H}, J_{4,3} 3.1 \mathrm{~Hz}\right.$, $\left.\mathrm{H}-4^{\mathrm{III}}\right), 5.96\left(\mathrm{~d}, 1 \mathrm{H}, \mathrm{H}-4^{\mathrm{IV}}\right), 7.25-8.10(\mathrm{~m}, 60 \mathrm{H}, \mathrm{Ph})$; ${ }^{13} \mathrm{C}$ NMR (100 MHz, $\left.\mathrm{CDCl}_{3}\right): 19.83,20.17,20.81,20.95$ $\left(4 \mathrm{COCH}_{3}\right), 55.20\left(\mathrm{OCH}_{3}\right), 61.39\left(\mathrm{C}-6^{\mathrm{IV}}\right), 61.46\left(\mathrm{C}-6^{\mathrm{II}}\right)$, $63.31\left(\mathrm{C}-5^{\mathrm{V}}\right), 63.52\left(\mathrm{C}-5^{\mathrm{VI}}\right), 65.68\left(\mathrm{C}-6^{\mathrm{III}}\right), 66.81\left(\mathrm{C}-6^{\mathrm{I}}\right)$, $67.75\left(\mathrm{C}-4^{\mathrm{IV}}\right), 68.88\left(\mathrm{C}-5^{\mathrm{I}}\right), 69.40\left(\mathrm{C}-5^{\mathrm{III}}\right), 69.76\left(\mathrm{C}-2^{\mathrm{III}}\right)$, $69.91\left(\mathrm{C}-2^{\mathrm{IV}}\right), 70.05\left(\mathrm{C}-2^{\mathrm{I}}\right), 70.17\left(\mathrm{C}-4^{\mathrm{III}}\right), 70.52\left(\mathrm{C}^{\mathrm{I}} \mathbf{I}^{\mathrm{I}}\right)$, $70.95\left(\mathrm{C}-5^{\mathrm{IV}}\right), 71.60\left(\mathrm{C}-3^{\mathrm{IV}}\right), 71.73\left(\mathrm{C}-5^{\mathrm{II}}\right), 71.92\left(\mathrm{C}-3^{\mathrm{II}}\right)$, $72.51\left(\mathrm{C}-2^{\mathrm{II}}\right), 73.55\left(\mathrm{C}-3^{\mathrm{I}}\right), 73.90\left(\mathrm{C}-3^{\mathrm{III}}\right), 77.51\left(\mathrm{C}-3^{\mathrm{V}}\right)$, $77.89\left(\mathrm{C}-3^{\mathrm{VI}}\right), 78.64\left(\mathrm{C}-4^{\mathrm{II}}\right), 80.30\left(\mathrm{C}^{\mathrm{V}} 4^{\mathrm{V}}\right), 81.24\left(\mathrm{C}-4^{\mathrm{VI}}\right)$, $81.84\left(\mathrm{C}-2^{\mathrm{V}}\right), 82.67\left(\mathrm{C}-2^{\mathrm{VI}}\right), 96.79\left(\mathrm{C}-1^{\mathrm{I}}\right), 98.50\left(\mathrm{C}-1^{\mathrm{III}}\right)$, $101.51\left(\mathrm{C}-1^{\mathrm{IV}}\right), 101.67\left(\mathrm{C}-1^{\mathrm{II}}\right), 106.06\left(\mathrm{C}-1^{\mathrm{V}}\right), 106.21$ $\left(\mathrm{C}-1^{\mathrm{VI}}\right), 128.18,128.21,128.26,128.35,128.39,128.44$, $128.47,128.54,129.51,129.67,129.72,129.78,129.84$, $129.92,130.01,133.45,165.36,165.49,165.52,165.66$, $165.69,165.74,166.15,169.74,169.91,170.14,170.32$; MALDITOF-MS: Calcd for $\mathrm{C}_{127} \mathrm{H}_{116} \mathrm{O}_{45}, 2360.68$ [M]; Found, $2383.32[\mathrm{M}+\mathrm{Na}]^{+}$. Anal. Calcd for $\mathrm{C}_{127} \mathrm{H}_{116^{-}}$ $\mathrm{O}_{45}$ : C, 64.57; H, 4.95. Found: C, 64.81; H, 5.06.

Methyl $\beta$-D-galactopyranosyl- $(1 \rightarrow 3)-[\alpha-\mathrm{L}$-arabinofuranosyl- $(1 \rightarrow 6)]-\alpha-\mathrm{D}$ - galactopyranosyl- $(1 \rightarrow 4)-\beta$ - Dgalactopyranosyl- $(1 \rightarrow 3)$-[ $\alpha$-L-arabinofuranosyl- $(1 \rightarrow 6)]-$ $\alpha$-D-galactopyranoside (23). - A solution of $22(0.27 \mathrm{~g}$, $0.114 \mathrm{mmol})$ in ammonia-saturated $\mathrm{MeOH}(100 \mathrm{~mL})$ was stirred at $\mathrm{rt}$ for 7 days. The solvents were evaporated, and the residue was purified on a Sephadex LH-20 column with $\mathrm{MeOH}$ as the eluent to give 23 as an amorphous solid $(0.10 \mathrm{~g}, 93 \%):[\alpha]_{\mathrm{D}}+7^{\circ}(c 1$, $\left.\mathrm{CH}_{3} \mathrm{OH}\right) ;{ }^{1} \mathrm{H}$ NMR $\left(400 \mathrm{MHz}, \mathrm{D}_{2} \mathrm{O}\right): 3.38(\mathrm{~s}, 3 \mathrm{H}$, $\left.\mathrm{OCH}_{3}\right), 3.61-4.10(\mathrm{~m}, 32 \mathrm{H}), 4.18(\mathrm{~d}, 1 \mathrm{H}, J 2.8 \mathrm{~Hz})$, $4.26(\mathrm{~d}, 1 \mathrm{H}, J 2.4 \mathrm{~Hz}), 4.60\left(\mathrm{~d}, 1 \mathrm{H}, J_{1,2} 7.6 \mathrm{~Hz}\right), 4.62$ $\left(\mathrm{d}, 1 \mathrm{H}, J_{1,2} 8.0 \mathrm{~Hz}\right), 4.81\left(1 \mathrm{H}\right.$, overlapped by $\left.\mathrm{D}_{2} \mathrm{O}\right)$, $4.96\left(\mathrm{~d}, 1 \mathrm{H}, J_{1,2} 3.6 \mathrm{~Hz}\right), 4.20(\mathrm{~s}, 1 \mathrm{H}), 5.20\left(\mathrm{~d}, 1 \mathrm{H}, J_{1,2}\right.$ $1.1 \mathrm{~Hz}) ;{ }^{13} \mathrm{C}$ NMR $\left(100 \mathrm{MHz}, \mathrm{D}_{2} \mathrm{O}\right): 56.03\left(\mathrm{OCH}_{3}\right)$, $58.42,61.07,61.80,62.01,67.20,68.06,68.21,68.43$, 69.42, 69.84, 70.02, 70.08, 71.91 (3 C), 71.96, 72.67, 73.04, 73.37, 75.69, 75.86, 77.23, 77.34, 78.62, 79.74, $80.20,81.64,81.90,84.62,84.67,100.16\left(\mathrm{C}-1^{\mathrm{I}}, J_{\mathrm{C}-1, \mathrm{H}-1}\right.$ $173 \mathrm{~Hz}), 101.10\left(\mathrm{C}-1^{\mathrm{III}}, J_{\mathrm{C}-1, \mathrm{H}-1} 171 \mathrm{~Hz}\right), 105.22\left(\mathrm{C}-1^{\mathrm{IV}}\right.$, $\left.J_{\mathrm{C}-1, \mathrm{H}-1} 164 \mathrm{~Hz}\right), 105.39\left(\mathrm{C}-1^{\mathrm{II}}, J_{\mathrm{C}-1, \mathrm{H}-1} 165 \mathrm{~Hz}\right), 108.24$ $\left(\mathrm{C}-1^{\mathrm{V}}, J_{\mathrm{C}-1, \mathrm{H}-1} 173 \mathrm{~Hz}\right), 108.72\left(\mathrm{C}-1^{\mathrm{VI}}, 174 \mathrm{~Hz}\right)$; ESI-MS Calcd for $\mathrm{C}_{83} \mathrm{H}_{72} \mathrm{O}_{23} \mathrm{~S}, 944.3[\mathrm{M}]$; Found, $962.6[\mathrm{M}+$ $\left.\mathrm{NH}_{4}\right]^{+}$.

\section{Acknowledgements}

This work was supported by National Nature Science Foundation of China (Projects 39970179 and 29972053).

\section{References}

1. Chinese Herbs and Compatibility; Chen, P. Ed.; Beijing: Science Press, 1997, p. 452.

2. The Pharmacopoeia Committee of PR China. Pharmacopoeia (I); Beijing: People's Health Press, 1995.

3. Fang, J. personal communication.

4. (a) Schmidt, R. R.; Kinzy, W. Adv. Carbohydr. Chem. Biochem. 1994, 50, 21-123;

(b) Toshima, K.; Tatsuta, K. Chem. Rev. 1993, 93, 15021531.

5. (a) Preparative Carbohydrate Chemistry; Hanessian, S. Ed.; New York: Marcel Dekker, 1996;

(b) Verduyn, R.; Douwes, M.; van der Klein, P. A. M.; Mosinger, E. M.; van der Marel, G. A.; van Boom, J. H. Tetrahedron 1993, 49, 7301-7316;

(c) Du, Y.; Zhang, M.; Kong, F. J. Chem. Soc., Perkin Trans. 1 2001, 2289-2293;

(d) Du, Y.; Zhang, M.; Kong, F. Tetrahedron 2001, 57, 1757-1763;

(e) Boons, G.-J.; Hale, K. J. Organic Synthesis with Carbohydrates; Sheffield Academic Press: UK, 2000.

6. Du, Y.; Pan, Q.; Kong, F. Carbohydr. Res. 2000, 323, 28-35.

7. Catelani, G.; Colonna, F.; Marra, A. Carbohydr. Res. 1988, 182, 297-300.

8. Pan, Q.; Du, Y.; Kong, F.; Pan, J.; Lu, M. J. Carbohydr. Chem. 2001, 20, 297-306.

9. Greene, T. W.; Wuts, P. G. M. Protective Groups in Organic Synthesis, 3rd ed.; New York: Wiley, 1999.

10. Fiandor, J.; Garcia-Lopez, M. T.; de las Heras, F. G.; Mendez-Castrillon, P. P. Synthesis 1985, 1121-1123.

11. Schmidt, R. R.; Michel, J. J. Carbohydr. Chem. 1985, 4, $141-169$. 